\title{
Probabilistic Inventory Model under Flexible Trade Credit Plan Depending upon Ordering Amount
}

\author{
Piyali Mallick ${ }^{1, *}$, Lakshmi Narayan De $^{2}$ \\ ${ }^{1}$ Department of Mathematics, Government General Degree College, Kharagpur-II, West Bengal, India \\ ${ }^{2}$ Department of Mathematics, Haldia Govt. College, Haldia, West Bengal, India
}

Received July 27, 2020; Revised August 31, 2020; Accepted September 29, 2020

\begin{abstract}
Cite This Paper in the following Citation Styles
(a): [1] Piyali Mallick, Lakshmi Narayan De, "Probabilistic Inventory Model under Flexible Trade Credit Plan Depending upon Ordering Amount," Mathematics and Statistics, Vol. 8, No. 5, pp. 596 - 609, 2020. DOI: $10.13189 / \mathrm{ms} .2020 .080515$.
\end{abstract}

(b): Piyali Mallick, Lakshmi Narayan De (2020). Probabilistic Inventory Model under Flexible Trade Credit Plan Depending upon Ordering Amount. Mathematics and Statistics, 8(5), 596 - 609. DOI: 10.13189/ms.2020.080515.

Copyright $\odot 2020$ by authors, all rights reserved. Authors agree that this article remains permanently open access under the terms of the Creative Commons Attribution License 4.0 International License

\begin{abstract}
In this work, we propose a stochastic inventory model under the situations that delay in imbursement is acceptable. Most of the inventory model on this topic supposed that the supplier would offer the retailer a fixed delay period and the retailer could sell the goods and accumulate revenue and earn interest with in the credit period. They also assumed that the trade credit period is independent of the order quantity. Limited investigators developed EOQ model under permissible delay in payments, where trade credit is connected with the order quantity. When the order quantity is a lesser amount of the quantity at which the delay in payment is not permitted, the payments for the items must be made immediately. Otherwise, the fixed credit period is permitted. However, all these models were completely deterministic in nature. In reality, this trade credit period cannot be fixed. If it is fixed, then retailer will not be interested to buy higher quantity than the fixed quantity at which delay in payment is permitted. To reflect this situation, we assumed that trade credit period is not static but fluctuates with the ordering quantity. The demand throughout any arrangement period follows a probability distribution. We have calculated the total variable cost for every unit of time. The optimum ordering policy of the scheme can be found with the aid of three theorems (proofs are provided). An algorithm to determine the best ordering rule with the assistance of the propositions is established and numerical instances are provided for clarification. Sensitivity investigation of all the parameters of the model is presented and deliberated. Some previously published results are special cases of the
\end{abstract}

consequences gotten in this paper.

Keywords Probabilistic Inventory Model, Trade Credit, Permissible Delay in Payments

\section{Introduction}

In developing traditional optimal ordering policy of an inventory model, it is generally assumed that the retailer must pay to supplier for the products at the time receiving of substances as every business owner would like to have all sales on a cash basis. However, in practice it is not always possible in competitive market place. Supplier allows retailer a certain a delay period (credit period) for settling down the account and no interest is charged on the unsettled account if the account is settled by the end of the credit period. The supplier will charge higher interest if the account is not settled within the trade credit period. Using this trade credit policy, suppliers can attract additional customers by not demanding cash up front. Trade credit can be advantageous for the new retailer incapable to raise capital or secure business loans, yet needs stock quickly. Using trade credit, business to be flexible, adapting to market demands and seasonal variations so that retailer has a constant supply of goods even when histher finances are not stable. Supplier can mix trade credit with bulk discounting to encourage buyers to speed more. Supplier's trade credit can prevent buyers from looking elsewhere and 
strengthen the supplier-buyer relationship. The most of suppliers frequently make exercise this plan to boost their commodities though there are some disadvantages of trade credit like late payment, cash flow problem, customer assessment, account handling etc. Goyal [10] first established an EOQ model under permissible delay in payments. In his model supplier allows a fixed time period for settling down the account, supplier is essentially giving his customer a loan without interest throughout this period. Chung et al. [8] settled a substitute method to determine the optimal ordering procedure under the condition of delay in payments. Shah and Shah [21] deliberated the same model by tolerating deficiencies. Shah and Shah [22] first considered a probabilistic model where delay in payment is tolerable. They assumed more realistic assumption that demand is not deterministic it follows probabilistic distribution. Shah et al. [24] developed the equivalent model, where time was treated as a continuous variable. In another paper, Shah and Shah [23] also established a discrete-time probabilistic inventory model under permitted delay in payments. Many scholars, such as Aggarwal and Jaggy [1], Hwang and Shinn [13], Jamal et al. [14], Sarker et al. [20], Huang [12], Mahato [17], Jiang Wu et al. [15], Musa and Sani [18], Li et al. [16] and Pramanick and Maity [19] also developed inventory models combining acceptable delay in payment into account. All inventory models cited above were made under the consideration that the trade credit plan is fixed. The extend and pattern of trade credit in an industry or business sector depend on a number of factors, including the average rate of turnover of stock, the nature of the goods involved - e.g. their perishability, the relative size of buying and selling firms, and the degree of competition. Several researchers made their work by assuming the fact that delay period is dependent on size of buying of the product. Chang et al. [2], Chung et al. [8], Chung et al. [5], Chang et al. [3], Chung et al. [7], Teng et al. [25] Chen et al. [4], Tiwari et al. [26] developed economic models under permitted delay in payment, where the trade credit period is connected to the order number. Once the order quantity is fewer than the amount at which the delay in payment is allowed, the payment for the matters must be made instantly. If not, a fixed trade credit is allowed. The supplier practices this strategy to encourage retailer to order an extra quantity. However, these aforementioned models were entirely deterministic in nature. In reality, this trade credit period cannot be fixed. If it is fixed, then the retailer will not be concerned in purchasing higher quantity than the fixed quantity at which delay in payment is permitted. To reflect this circumstance, an inventory model is settled under the assumption that the trade credit period is not only allied to ordering quantity but also fluctuates with the ordering quantity. It is also supposed that the demand is a continuous random variable following some probabilistic distribution. As it is seen in paper of De and Goswami [10] that continuous cycle time produces better result than discrete, so in this paper only continuous cycle time is considered. It is also showed that the optimal ordering strategy can be determined by means of our Theorems 1, 2 and 3. Outcomes found in this paper are exemplified with the support of a set of numerical examples and sensitiveness of different parameters are also contained within.

\section{Assumption and Natation}

Our proposed inventory model is framed with the following conventions and notations:

a). Time period is infinite i.e., there is no restriction for continuation of cycle.

b). Length of time between two successive orders is $T$, which is known as cycle time.

c). Items in inventory of the system are reviewed regularly at time interval $T$ between two successive orders, which is the fixed. At the termination of each interval of length $T$, items are ordered so as to bring the on-hand inventory level to a level $Q$.

d). In the time interval $T$, demand $x$ follows a probability density function (p.d.f.) $f(x \mid T)$, $a(T) \leq x \leq b(T) \quad$ with $\quad \mu(T)=E(x \mid T \quad)$ $=\int_{a(T)}^{b(T)} x f(x \mid T) d x=R T$ (say) (1) in continuous sense, where $\mu(T)$ is the mean demand during $T$ and $R=\frac{\mu(T)}{T}$ denotes the average expected demand per unit time during a cycle. It is also assumed that the p.d.f. $f(x \mid T)$ of the demand $x$ during $T$ is adequately well performed so as to all the expected costs discussed below exist. Correspondingly, the distribution of the demand is expected to be fixed over the planning horizon $T$.

e). In the procedure of obtaining the definite result, it is assumed that the forms of the maximum annual demand $b(T)$ as $(T)=P R T$, where $P \geq 1$ is a known constant.

f). Replenishment or renewal rate is infinite. Lead-time is zero. Shortages are not acceptable.

g). Supplier offers delay period when number of ordering quantity is greater or equal to $W$.

h). $A, C, S$ and $H$ are the cost for placing per order, unit buying cost per item, unit retailing/selling cost per item and unit stock holding cost per item per unit time respectively and are known constants. It is also presumed that $S \geq C$.

i). To encourage retailer to buy bigger substances or amount, it is supposed that if the retailer buys products from supplier fewer than a fixed amount $W$ (say) then the retailer will not get some facilities such as delay in payment. Consequently, delay period is increasing function of $Q$. For easiness, in this paper, it is assumed that the delay period is linearly dependent on ordering quantity. i.e., if $Q \geq W$, a variable credit period $M\left(M_{0}+\alpha Q ; \alpha \in[0,1]\right)$, is allowed; else a 
delay in payment is not permitted. The motives behind for selecting such value of $\alpha$ is that, if $\alpha<0$ then $M_{0}+\alpha Q$ will be a decreasing function of $Q$ which is unrealistic supposition. If $\alpha>1$ then the delay period will be so high that the supplier may face some problem to capitalize his personal turnover. $\mathrm{He}$ will face cash flow problem. So, it is assumed. But, normally $\alpha$ should be in $[0, a]$, where $a$ is close to 0 and less than 1.

j). The supplier provides a fixed credit period $M$ to settle the accounts to the retailer and the retailer, in turn, also off ers a credit period $N$ to each of its customers to settle the accounts, where $M \geq N$.

k). When the retailer must pay the amount of buying cost to the supplier, the retailer will borrow $100 \%$ purchasing cost from the bank to pay back the account with rate $I_{p}$. When $T \geq M$, the retailer returns money to the bank at the termination of the inventory cycle. However, when $\leq M$, the retailer returns money to the bank at $T=M$.

1). If the credit period is shorter than the cycle time, the retailer can sell the items, gather sales revenue and receives interest with rate $I_{e}$ all over the inventory cycle, where $I_{p} \geq I_{e}$.

m). $\operatorname{TVC}(T)$, a function of $T$, is the total relevant cost and $T^{*}$ is the optimal cycle time.

\section{Model Formulation}

The differential equation describing the inventory position $Q_{x}(t)(0 \leq t \leq T)$ of the system during the scheduling period $T$ is

$$
\frac{d Q_{x}(t)}{d t}=-\frac{x}{T}
$$

Using the boundary condition $Q_{x}(0)=Q$, the solution of equation (2) is

$$
Q_{x}(t)=Q-\frac{x}{T} t, 0 \leq t \leq T
$$

Since shortages are not permissible, using the state $Q_{x}(T)=0$ when $x=b(T)$,

$$
\text { we find } Q=b(T)
$$

By means of equation (4), (3) turns into

$$
Q_{x}(t)=b(T)-\frac{x}{T} t
$$

The average expected inventory in the system for every unit time is $\frac{1}{T} \int_{0}^{T} E\left(Q_{x}(t)\right) d t=(2 P-1) \frac{R T}{2}$.

The total annual variable cost involves the following elements. Two circumstances may arise.
I. $\frac{W}{P R} \leq M=M_{0}+\alpha P R T$
II. $\frac{W}{P R}>M=M_{0}+\alpha P R T$

Case I: $\frac{W}{P R} \leq M=M_{0}+\alpha P R T$.

(a) Ordering cost per unit time $=\frac{A}{T}$.

(b) Stock holding cost per unit time $(2 P-1) \frac{R T H}{2}$.

(c) Now according to the norms, three probable cases can happen specifically $0<T<\frac{W}{P R}, \frac{W}{P R} \leq T \leq M$ and $T \geq M$. These three cases are treated distinctly which are discussed below.

\section{Case (i) $0<T<\frac{W}{P R}$}

Expected interest payable per unit time $=\frac{C Q T I P}{T}=$ CIPPRT . Expected interest earned per unit time = $\frac{\text { SIe }}{T} \int_{0}^{T} E\left(\frac{x}{T}\right) t d t=\frac{R T S I e}{2}$.

\section{Case (ii) $\frac{W}{P R} \leq T \leq M$}

Expected interest payable per unit time $=0$. Expected interest earned per unit time $=\frac{S I e}{T}\left[\frac{R T^{2}}{2}+R T(M-T)\right]=$ RSIe $\left[M_{0}+\alpha P R T-\frac{T}{2}\right]$.

\section{Case (iii) $T \geq M=M_{0}+\alpha P R T$}

Expected interest payable per unit time $=\frac{C Q(T-M) I p}{T}$ $=\frac{C P R(T-M 0-\alpha P R T) I p}{T}$.

Expected interest earned per unit time = $\frac{S I e}{T} \int_{0}^{T} E\left(\frac{x}{T}\right) t d t=\frac{R T S I e}{2}$.

From the above arguments, the appropriate total cost per unit time for the retailer can be stated as

$$
T V C(T)=\left\{\begin{array}{c}
T V C_{1}(T), \quad \text { if } 0<T<\frac{W}{P R} \\
T V C_{2}(T), \text { if } \frac{W}{P R} \leq T \leq M_{0}+\alpha P R T \\
T V C_{3}(T), \text { if } M_{0}+\alpha P R T \leq T
\end{array}\right.
$$

Where,

$$
\begin{aligned}
& T V C_{1}(T)=\frac{A}{T}+(2 P-1) \frac{R T H}{2}+C I P P R T-\frac{R T S I e}{2} \\
& T V C_{2}(T)=\frac{A}{T}+(2 P-1) \frac{R T H}{2}-R S I e\left[M_{0}+\alpha P R T-\frac{T}{2}\right]
\end{aligned}
$$

$T V C_{3}(T)=\frac{A}{T}+(2 P-1) \frac{R T H}{2}+\frac{C P R(T-M 0-\alpha P R T) I p}{T}-\frac{R T S I e}{2}$

All $T V C_{1}(T), T V C_{2}(T)$, and $T V C_{3}(T)$ are defined on $T>0$.

Equations (7)-(9) produce

$$
T V C_{1}^{\prime}(T)=-\frac{A}{T^{2}}+\frac{R(H(2 P-1)+2 C I p P-S I e)}{2}
$$




$$
\begin{gathered}
T V C_{1}^{\prime \prime}(T)=\frac{2 A}{T^{3}}>0 \\
T V C_{2}^{\prime}(T)=-\frac{A}{T^{2}}+\frac{R\left(H(2 P-1)-2 S I_{e} \alpha P R+S I e\right)}{2} \\
T V C_{2}^{\prime \prime}(T)=\frac{2 A}{T^{3}}>0 \\
T V C_{3}^{\prime}(T)=-\frac{A}{T^{2}}+\frac{R\left(H(2 P-1)+2 C I p P-2 C I_{p} \alpha P^{2} R-S I e\right)}{2} \\
T V C_{3}^{\prime \prime}(T)=\frac{2 A}{T^{3}}>0
\end{gathered}
$$

Equations (11), (13) and (15) imply that $T V C_{1}(T)$, $T V C_{2}(T)$ and $T V C_{3}(T)$ are convex for $T>0$.

Case II: $\frac{W}{P R}>M=M_{0}+\alpha P R T$.

In this case equation (6) can be written as follows:

$$
T V C(T)=\left\{\begin{array}{l}
T V C_{1}(T), \text { if } 0<T<\frac{W}{P R} \\
T V C_{3}(T), \quad \text { if } T \geq \frac{W}{P R}
\end{array}\right.
$$

Here $T V C(T)$ is continuous except at $T=\frac{W}{P R}$.

Now solving $T V C_{i}^{\prime}(T)=0$ for $=1,2,3$, we obtain

$$
\begin{gathered}
T_{1}^{*}=\sqrt{\frac{2 A}{R(H(2 P-1)+2 C I P P-S I e)}} \text { if } R(H(2 P-1)+ \\
T_{2}^{*}=\sqrt{\frac{2 C I p P-S I e)>0}{R\left(H(2 P-1)-2 S I_{e} \alpha P R+S I e\right)}} \text { if } R(H(2 P-1)- \\
T_{3}^{*}=\sqrt{\frac{\left.2 S I_{e} \alpha P R+S I e\right)>0}{R\left(H(2 P-1)+2 C I P P-2 C I_{p} \alpha P^{2} R-S I e\right)}} \text { if } R(H(2 P- \\
\left.1)+2 C I p P-2 C I_{p} \alpha P^{2} R-S I e\right)>0
\end{gathered}
$$
that

By the convexity of $T V C_{i}(T)(i=1,2,3)$, it is detected

$$
T V C_{i}^{\prime}(T)= \begin{cases}<0, & \text { if } T<T_{i}^{*} \\ =0, & \text { if } T=T_{i}^{*} \\ >0, & \text { if } T>T_{i}^{*}\end{cases}
$$

\section{Decision Rule of the Optimal Cycle Time When $\frac{W}{P R} \leq M=M_{0}+\alpha P R T$}

In this case two possibilities may arise namely $\alpha P R \geq$ 1 and $R<1$. These two cases are treated separately which are discussed below

\section{Case (i) $\alpha P R \geq 1$}

Here $T V C(T)$ will be modified as (since $\alpha P R \geq 1$ and so $T$ can be grater than or equal to $\left.M_{0}+\alpha P R T\right)$

$$
\operatorname{TVC}(T)=\left\{\begin{array}{l}
T V C_{1}(T), \text { if } 0<T<\frac{W}{P R} \\
T V C_{2}(T), \quad \text { if } T \geq \frac{W}{P R}
\end{array}\right.
$$

Here also $T V C(T)$ is continuous except at $T=\frac{W}{P R}$.

In this case Equations (10) and (12) yield

$$
\begin{gathered}
T_{1}^{*} \geq \frac{W}{P R} \text { implies } T V C_{1}^{\prime}\left(\frac{W}{P R}\right) \leq 0 \text { and hence } T V C_{1}(T) \text { is } \\
\text { decreasing on }\left(0, \frac{W}{P R}\right)
\end{gathered}
$$

$$
T_{2}^{*}<\frac{W}{P R} \text { implies } T V C_{2}^{\prime}\left(\frac{W}{P R}\right)>0 \text { and hence } T V C_{2}(T) \text { is }
$$

$$
\text { increasing on }\left[\frac{W}{P R}, \infty\right)
$$

Furthermore, it follows the result

Theorem 1. (A) Suppose that $H(2 P-1)-$ $2 S I_{e} \alpha P R+S I e<0$ then $T^{*}=\infty$ and $\operatorname{TVC}\left(T^{*}\right)=$ $-\infty$ ie., the retailer will try to continue his cycle as much as possible.

Proof: If $H(2 P-1)-2 S I_{e} \alpha P R+S I e<0$, Equation (12) implies that $\operatorname{TVC}(T)$ is decreasing for $T \geq \frac{W}{P R}$. Since $\lim _{T \rightarrow \infty} T V C(T)=-$ RSIe $M_{0}+\lim _{T \rightarrow \infty} \frac{R T}{2}(H(2 P-1)-$ 2 SIe $\alpha P R+S I e)=-\infty$ and $\lim _{T \rightarrow 0^{+}} \operatorname{TVC}(T)=\infty$ so we conclude that $T^{*}=\infty$ and $\operatorname{TVC}\left(T^{*}\right)=-\infty$

(B) Suppose that $H(2 P-1)-2 S I_{e} \alpha P R+S I e=0$ then $R\left(H(2 P-1)+2 C I_{p} P-S I e>0\right.$ (since $\alpha P R \geq$ 1) and

(a) If $T_{1}^{*} \geq \frac{W}{P R}$, then $T^{*}=\infty$ and $\operatorname{TVC}\left(T^{*}\right)=$ - RSIeM $M_{0}$

(b) If $T_{1}^{*}<\frac{W}{P R}$, then $\operatorname{TVC}\left(T^{*}\right)=\min \left[T V C_{1}\left(T_{1}^{*}\right)-\right.$ RSIe $\left.M_{0}\right]$ and $T^{*}=T_{1}^{*}$ or $\infty$ associated with the least cost).

Proof: (a) If $H(2 P-1)-2 S I_{e} \alpha P R+S I e=0$ and $T_{1}^{*} \geq \frac{W}{P R}$ then equation (12) and (17) imply that $\operatorname{TVC}(T)$ is decreasing on $(0, \infty)$. Consequently $T^{*}=$ $\infty$ and $\operatorname{TVC}\left(T^{*}\right)=\infty$.

(b) If $H(2 P-1)-2 S I_{e} \alpha P R+S I e=0$ and $T_{1}^{*}<\frac{W}{P R}$, then equation (12) and (17) imply that $T V C(T)$ is decreasing on $\left(0, T_{1}^{*}\right)$, increasing on $\left[T_{1}^{*}, \frac{W}{P R}\right)$ and decreasing on $\left[\frac{W}{P R}, \infty\right)$. Hence $T^{*}=T_{1}^{*}$ or $\infty$ associated with the least cost) and $\operatorname{TVC}\left(T^{*}\right)=\min \left[T V C_{1}\left(T_{1}^{*}\right)-\right.$ RSIe $\left.M_{0}\right]$.

(C) Suppose that $H(2 P-1)-2 S I_{e} \alpha P R+S I e>0$ then $H(2 P-1)+2 C I_{p} P-$ SIe $>0($ since $\alpha P R \geq 1)$ and 
(a) If $T_{1}^{*}<\frac{W}{P R}, T_{2}^{*}<\frac{W}{P R}$ then $T^{*}=T_{1}^{*}$ and $\operatorname{TVC}\left(T^{*}\right)=T V C_{1}\left(T_{1}^{*}\right)$.

(b) If $T_{1}^{*}<\frac{W}{P R}, T_{2}^{*} \geq \frac{W}{P R}$ then $T^{*}=T_{1}^{*} \quad$ or $T_{2}^{*}$ (associated with the least cost) and $\operatorname{TVC}\left(T^{*}\right)=\min$ $\left[T V C_{1}\left(T_{1}^{*}\right), T V C_{2}\left(T_{2}^{*}\right)\right]$.

(c) If $T_{1}^{*} \geq \frac{W}{P R}, T_{2}^{*}<\frac{W}{P R} \quad$ then $\quad T^{*}=\frac{W}{P R} \quad$ and $\operatorname{TVC}\left(T^{*}\right)=T V C_{2}\left(\frac{W}{P R}\right)$.

(d) If $\quad T_{1}^{*} \geq \frac{W}{P R}, T_{2}^{*} \geq \frac{W}{P R} \quad$ then $\quad T^{*}=T_{2}^{*} \quad$ and $\operatorname{TVC}\left(T^{*}\right)=T V C_{2}\left(T_{2}^{*}\right)$.

Proof: (a) If $T_{1}^{*}<\frac{W}{P R}, T_{2}^{*}<\frac{W}{P R}$ then Equations (17) and (20) imply that $\operatorname{TVC}(T)$ is decreasing on $\left(0, T_{1}^{*}\right]$, increasing on $\left[T_{1}^{*}, \frac{W}{P R}\right)$ and decreasing on $\left[\frac{W}{P R}, \infty\right)$. Consequently $T^{*}=T_{1}^{*}$ and $\operatorname{TVC}\left(T^{*}\right)=T V C_{1}\left(T_{1}^{*}\right)$.

(b) If $T_{1}^{*}<\frac{W}{P R}, T_{2}^{*} \geq \frac{W}{P R}$ then (17) implies that $\operatorname{TVC}(T)$ is decreasing on $\left(0, T_{1}^{*}\right]$, increasing on $\left[T_{1}^{*}, \frac{W}{P R}\right)$, decreasing on $\left[\frac{W}{P R}, T_{2}^{*}\right]$ and increasing on $\left[T_{2}^{*}, \infty\right)$. Consequently, $T^{*}=T_{1}^{*}$ or $T_{2}^{*}$ (associated with the least cost) and $\operatorname{TVC}\left(T^{*}\right)=\min \left[T V C_{1}\left(T_{1}^{*}\right), T V C_{2}\left(T_{2}^{*}\right)\right]$.

(c) If $T_{1}^{*} \geq \frac{W}{P R}, T_{2}^{*}<\frac{W}{P R}$ then Equations (19) and (20) imply that $\operatorname{TVC}(T)$ is decreasing on $\left(0, \frac{W}{P R}\right)$ and increasing on $\left[\frac{W}{P R}, \infty\right)$. Consequently $T^{*}=\frac{W}{P R}$ and $\operatorname{TVC}\left(T^{*}\right)=T V C_{2}\left(\frac{W}{P R}\right)$.

(d) If $T_{1}^{*} \geq \frac{W}{P R}, T_{2}^{*} \geq \frac{W}{P R}$ then Equations (19) and (17) imply that $T V C(T)$ is decreasing on $\left(0, \frac{W}{P R}\right)$, decreasing on $\left[\frac{W}{P R}, T_{2}^{*}\right]$, and increasing on $\left[T_{2}^{*}, \infty\right)$.Consequently $T^{*}=T_{2}^{*}$ and $\operatorname{TVC}\left(T^{*}\right)=T V C_{2}\left(T_{2}^{*}\right)$.

\section{Case (ii) $\alpha P R<1$}

Here $\operatorname{TVC}(T)$ will be modified as $\operatorname{TVC}(T)=$ $\left\{\begin{array}{lr}T V C_{1}(T), \quad \text { if } 0<T<\frac{W}{P R} \\ T V C_{2}(T), \quad \text { if } \frac{W}{P R} \leq T \leq \frac{M_{0}}{1-\alpha P R} \\ T V C_{3}(T), \quad \text { if } \frac{M_{0}}{1-\alpha P R} \leq T\end{array}\right.$

In this case Equations (10), (12) and (14) yield that

$T_{1}^{*} \geq \frac{W}{P R}$ implies $T V C_{1}^{\prime}\left(\frac{W}{P R}\right) \leq 0$ and hence $T V C_{1}(T)$ is decreasing on $\left(0, \frac{W}{P R}\right)$

$T_{2}^{*}<\frac{W}{P R}$ implies $T V C_{2}^{\prime}\left(\frac{W}{P R}\right)>0$ and hence $T V C_{2}(T)$ is increasing on $\left[\frac{W}{P R}, \frac{M_{0}}{1-\alpha P R}\right]$

$T_{2}^{*}>\frac{M_{0}}{1-\alpha P R}$ implies $T V C_{2}^{\prime}\left(\frac{M_{0}}{1-\alpha P R}\right)<0$ and hence
$T V C_{2}(T)$ is decreasing on $\left[\frac{W}{P R}, \frac{M_{0}}{1-\alpha P R}\right]$

$T_{3}^{*}<\frac{M_{0}}{1-\alpha P R}$ implies $T V C_{3}^{\prime}\left(\frac{M_{0}}{1-\alpha P R}\right)>0$ and hence

$T V C_{3}(T)$ is increasing on $\left[\frac{M_{0}}{1-\alpha P R}, \infty\right)$

Furthermore, the result follows.

Theorem 2. (A) Suppose that $H(2 P-1)+2 C I P P-$ $2 C I_{p} \alpha P^{2} R-S I e<0$ then $T^{*}=\infty$ and $\operatorname{TVC}\left(T^{*}\right)=$ $\infty$.ie., the retailer will try to continue his cycle as much as possible.

Proof. If $H(2 P-1)+2 C I_{p} P-2 C I_{p} \alpha P^{2} R-S I_{e}<$ 0 , then equations (14) and (21) imply that $\operatorname{TVC}(T)$ is decreasing for $T \geq \frac{M_{0}}{1-\alpha P R}$. Since $\lim _{T \rightarrow \infty} \operatorname{TV} C(T)=$ $-C I_{p} P R M_{0}+\lim _{T \rightarrow \infty} \frac{R T}{2}\left(H(2 P-1)-2 S I_{e} \alpha P R+S I e\right)=$ $-\infty$ and $\lim _{T \rightarrow 0^{+}} \operatorname{TVC}(T)=\infty$ so $T^{*}=$ $\infty$ and $\operatorname{TVC}\left(T^{*}\right)=\infty$.

(B) Suppose that $H(2 P-1)+2 C I_{p} P-$ $2 C I_{p} \alpha P^{2} R-S I_{e}=0$ then

(i) If $H(2 P-1)+2 C I_{p} P-S I_{e}=0$ and $H(2 P-$ 1) $-2 S I_{e} \alpha P R+S I e \leq 0$ then $T^{*}=$ $\infty$ and $\operatorname{TVC}\left(T^{*}\right)=-C I_{p} P R M_{0}$.

(ii) If $H(2 P-1)+2 C I_{p} P-S I_{e}=0$ and $H(2 P-$ 1) $-2 S I_{e} \alpha P R+S I e>0$ then

(a) If $T_{2}^{*}>\frac{M_{0}}{1-\alpha P R}$ then $T^{*}=\infty$ and $\operatorname{TVC}\left(T^{*}\right)=$ $-C I_{p} P R M_{0}$.

(b) If $\frac{W}{P R} \leq T_{2}^{*} \leq \frac{M_{0}}{1-\alpha P R}$ then $T^{*}=T_{2}^{*}$ or $\infty$ (associated with the least cost) $\operatorname{TVC}\left(T^{*}\right)=\min \left[\operatorname{TVC}_{2}\left(T_{2}^{*}\right)\right.$, $\left.-C I_{p} P R M_{0}\right]$.

(c) If $T_{2}^{*}<\frac{W}{P R}$ then $T^{*}=\frac{W}{P R}$ or $\infty$ (associated with the least cost) $T V C\left(T^{*}\right)=\min \left[T V C_{2}\left(\frac{W}{P R}\right),-C I_{p} P R M_{0}\right]$.

(iii) If $H(2 P-1)+2 C I_{p} P-S I_{e}>0$ and $H(2 P-$ 1) $-2 S I_{e} \alpha P R+S I_{e} \leq 0$ then

(a) If $T_{1}^{*} \geq \frac{W}{P R}$ then $T^{*}=\infty$ and $\operatorname{TVC}\left(T^{*}\right)=$ $-C I_{p} P R M_{0}$.

(b) If $T_{1}^{*}<\frac{W}{P R}$ then $T^{*}=T_{1}^{*}$ or $\infty$ (associated with the least cost) $\operatorname{TVC}\left(T^{*}\right)=\min \left[T V C_{1}\left(T_{1}^{*}\right),-C I_{p} P R M_{0}\right]$.

(iv) If $H(2 P-1)+2 C I_{p} P-S I_{e}>0$ and $H(2 P-$ 1) $-2 S I_{e} \alpha P R+S I_{e}>0$ then

(a) If $T_{1}^{*} \geq \frac{W}{P R}$ and $T_{2}^{*}>\frac{M_{0}}{1-\alpha P R}$ then $T^{*}=$ $\infty$ and $\operatorname{TVC}\left(T^{*}\right)=-C I_{p} P R M_{0}$.

(b) If $T_{1}^{*} \geq \frac{W}{P R}$ and $\frac{W}{P R} \leq T_{2}^{*} \leq \frac{M_{0}}{1-\alpha P R}$ then $T^{*}=T_{2}^{*}$ or 
$\infty$ (associated with the least $\cos t) \operatorname{TVC}\left(T^{*}\right)=\min$ $\left[T V C_{2}\left(T_{2}^{*}\right),-C I_{p} P R M_{0}\right]$.

(c) If $T_{1}^{*} \geq \frac{W}{P R}$ and $T_{2}^{*}<\frac{W}{P R}$ then $T^{*}=\frac{W}{P R}$ or $\infty$ (associated with the least cost) $\operatorname{TVC}\left(T^{*}\right)=\min$ $\left[T V C_{2}\left(\frac{W}{P R}\right),-C I_{p} P R M_{0}\right]$.

(d) If $T_{1}^{*}<\frac{W}{P R}$ and $T_{2}^{*}>\frac{M_{0}}{1-\alpha P R}$ then $T^{*}=T_{1}^{*}$ or $\infty$ (associated with the least cost) $\operatorname{TVC}\left(T^{*}\right)=\min \left[T V C_{1}\left(T_{1}^{*}\right)\right.$, $\left.-C I_{p} P R M_{0}\right]$.

(e) If $T_{1}^{*}<\frac{W}{P R}$ and $\frac{W}{P R} \leq T_{2}^{*} \leq \frac{M_{0}}{1-\alpha P R}$ then $T^{*}=T_{2}^{*}$, $T_{1}^{*}$ or $\infty$ (associated with the least cost) ) $T V C\left(T^{*}\right)=\min$ $\left[T V C_{1}\left(T_{1}^{*}\right), T V C_{2}\left(T_{2}^{*}\right),-C I_{p} P R M_{0}\right]$.

(f) If $T_{1}^{*}<\frac{W}{P R}$ and $T_{2}^{*}<\frac{W}{P R}$ then $T^{*}=T_{1}^{*}, \frac{W}{P R}$ or $\infty$ (associated with the least cost) $\operatorname{TVC}\left(T^{*}\right)=\min \left[T V C_{1}\left(T_{1}^{*}\right)\right.$, $\left.T V C_{2}\left(\frac{W}{P R}\right),-C I_{p} P R M_{0}\right]$.

Proof: (i) If $H(2 P-1)+2 C I_{p} P-2 C I_{p} \alpha P^{2} R-$ $S I_{e}=0, H(2 P-1)+2 C I_{p} P-S I_{e}=0$ and $H(2 P-$ 1) $-2 S I_{e} \alpha P R+S I e \leq 0$ then Equations (10), (12) and (14) imply that $\operatorname{TVC}(T)$ is decreasing on $(0, \infty)$. Since $\quad \lim _{T \rightarrow \infty} T V C(T)=-C I_{p} P R M_{0}+\lim _{T \rightarrow \infty} \frac{R T}{2}(H(2 P-$ 1) $\left.-2 S I_{e} \alpha P R+S I e\right)=-C I_{p} P R M_{0}$ and $\lim _{T \rightarrow 0^{+}} T V C(T)=$ $\infty$ so $T^{*}=\infty$ and $\operatorname{TVC}\left(T^{*}\right)=-C I_{p} P R M_{0}$.

(ii)(a) If $H(2 P-1)+2 C I_{p} P-2 C I_{p} \alpha P^{2} R-S I_{e}=0$, $H(2 P-1)+2 C I_{p} P-S I_{e}=0$ and $T_{2}^{*}>\frac{M_{0}}{1-\alpha P R}$ then Equations (10),(14) and (24) imply that $\operatorname{TVC}(T)$ is decreasing on $(0, \infty)$. Consequently $T^{*}=$ $\infty$ and $\operatorname{TVC}\left(T^{*}\right)=-C I_{p} P R M_{0}$

(b) If $H(2 P-1)+2 C I_{p} P-2 C I_{p} \alpha P^{2} R-S I_{e}=0$, $H(2 P-1)+2 C I_{p} P-S I_{e}=0$ and $\frac{W}{P R} \leq T_{2}^{*} \leq$ $\frac{M_{0}}{1-\alpha P R}$ then Equation (10),(14) and (17) imply that $T V C(T)$ is decreasing on $\left(0, T_{2}^{*}\right]$, increasing on $\left[T_{2}^{*}, \frac{M_{0}}{1-\alpha P R}\right]$ and decreasing on $\left[\frac{M_{0}}{1-\alpha P R}, \infty\right)$. Consequently $T^{*}=T_{2}^{*}$ or $\infty$ (associated with the least cost) $\operatorname{TVC}\left(T^{*}\right)=\min \left[T V C_{2}\left(T_{2}^{*}\right)\right.$, $\left.-C I_{p} P R M_{0}\right]$.

(c) If $H(2 P-1)+2 C I_{p} P-2 C I_{p} \alpha P^{2} R-S I_{e}=0$, $H(2 P-1)+2 C I_{p} P-S I_{e}=0$ and $T_{2}^{*}<\frac{W}{P R}$ then Equations (10), (14) and (23) imply that $\operatorname{TVC}(T)$ is decreasing on $\left(0 \frac{W}{P R}\right]$, increasing on $\left[\frac{W}{P R}, \frac{M_{0}}{1-\alpha P R}\right]$ and decreasing on $\left[\frac{M_{0}}{1-\alpha P R}, \infty\right)$. Consequently $T^{*}=\frac{W}{P R}$ or $\infty$ (associated with the least cost) $\operatorname{TVC}\left(T^{*}\right)=\min \left[T V C_{2}\left(\frac{W}{P R}\right)\right.$, $\left.-C I_{p} P R M_{0}\right]$.

(iii) (a)If $H(2 P-1)+2 C I_{p} P-S I_{e}>0, H(2 P-$ 1) $-2 S I_{e} \alpha P R+S I e \leq 0$ and $T_{1}^{*} \geq \frac{W}{P R}$ then Equations
(12),(14) and (22) imply that $T V C(T)$ is decreasing on (0, $\infty)$. Consequently $T^{*}=\infty$ and $\operatorname{TVC}\left(T^{*}\right)=-C I_{p} P R M_{0}$.

(b) If $H(2 P-1)+2 C I_{p} P-S I_{e}>0, H(2 P-1)-$ $2 S I_{e} \alpha P R+S I e \leq 0$ and $T_{1}^{*}<\frac{W}{P R}$ then Equations (12),(14) and (17) imply that $\operatorname{TVC}(T)$ is decreasing on $\left(0, T_{1}^{*}\right]$, increasing on $\left(T_{1}^{*}, \frac{W}{P R}\right)$ and decreasing on $\left[\frac{W}{P R}, \infty\right)$. Consequently $T^{*}=T_{1}^{*}$ or $\infty$ (associated with the least cost) $\operatorname{TVC}\left(T^{*}\right)=\min \left[T V C_{1}\left(T_{1}^{*}\right),-C I_{p} P R M_{0}\right]$.

(iv)(a) If $H(2 P-1)+2 C I_{p} P-S I_{e}>0, H(2 P-1)-$ $2 S I_{e} \alpha P R+S I e>0, \quad T_{1}^{*} \geq \frac{W}{P R}$ and $T_{2}^{*}>\frac{M_{0}}{1-\alpha P R}$ then Equations (14), (22) and (24) imply that $T V C(T)$ is decreasing on $[0, \infty)$. Consequently $T^{*}=$ $\infty$ and $\operatorname{TVC}\left(T^{*}\right)=-C I_{p} P R M_{0}$.

(b) If $H(2 P-1)+2 C I_{p} P-S I_{e}>0, H(2 P-1)-$ $2 S I_{e} \alpha P R+S I e>0, T_{1}^{*} \geq \frac{W}{P R}$ and $\frac{W}{P R} \leq T_{2}^{*} \leq \frac{M_{0}}{1-\alpha P R}$ then Equations (14), (22) and (17) imply that $\operatorname{TVC}(T)$ is decreasing on $\left(0, T_{2}^{*}\right]$, increasing on $\left[T_{2}^{*}, \frac{M_{0}}{1-\alpha P R}\right]$ and decreasing on $\left[\frac{M_{0}}{1-\alpha P R}, \infty\right)$. Consequently $T^{*}=T_{2}^{*}$ or $\infty$ (associated with the least cost) $\operatorname{TVC}\left(T^{*}\right)=\min$ $\left[T V C_{2}\left(T_{2}^{*}\right),-C I_{p} P R M_{0}\right]$.

(c) If $H(2 P-1)+2 C I_{p} P-S I_{e}>0, H(2 P-1)-$ $2 S I_{e} \alpha P R+S I e>0, T_{1}^{*} \geq \frac{W}{P R}$ and $T_{2}^{*}<\frac{W}{P R}$ then Equations (14), (22) and (23) imply that $T V C(T)$ is decreasing on (0, $\left.\frac{W}{P R}\right)$, increasing on $\left[\frac{W}{P R}, \frac{M_{0}}{1-\alpha P R}\right]$ and decreasing on $\left[\frac{M_{0}}{1-\alpha P R}\right.$, $\infty$ ). Consequently $T^{*}=\frac{W}{P R}$ or $\infty$ (associated with the least cost) $\operatorname{TVC}\left(T^{*}\right)=\min \left[T V C_{2}\left(\frac{W}{P R}\right),-C I_{p} P R M_{0}\right]$.

(d) If $H(2 P-1)+2 C I_{p} P-S I_{e}>0, H(2 P-1)-$ $2 S I_{e} \alpha P R+S I e>0, T_{1}^{*}<\frac{W}{P R}$ and $T_{2}^{*}>\frac{M_{0}}{1-\alpha P R}$ then Equations (14), (17) and (24) imply that $T V C(T)$ is decreasing on $\left(0, T_{1}^{*}\right]$, increasing on $\left[T_{1}^{*}, \frac{W}{P R}\right)$ and decreasing on $\left[\frac{W}{P R}, \infty\right)$. Consequently $T^{*}=T_{1}^{*}$ or $\infty$ (associated with the least cost) $\operatorname{TVC}\left(T^{*}\right)=\min \left[T V C_{1}\left(T_{1}^{*}\right)\right.$, $\left.-C I_{p} P R M_{0}\right]$.

(e) If $H(2 P-1)+2 C I_{p} P-S I_{e}>0, H(2 P-1)-$ $2 S I_{e} \alpha P R+S I e>0, T_{1}^{*}<\frac{W}{P R}$ and $\frac{W}{P R} \leq T_{2}^{*} \leq \frac{M_{0}}{1-\alpha P R}$ then Equations (14)and (17) imply that TVC (T) is decreasing on $\left(0, T_{1}^{*}\right]$, increasing on $\left[T_{1}^{*}, \frac{W}{P R}\right)$, decreasing on $\left[\frac{W}{P R}, T_{2}^{*}\right]$, increasing on $\left[T_{2}^{*}, \frac{M_{0}}{1-\alpha P R}\right]$ and decreasing on $\left[\frac{M_{0}}{1-\alpha P R}, \infty\right)$. Consequently $T^{*}=T_{2}^{*}, T_{1}^{*}$ or $\infty$ (associated with the least cost) $\operatorname{TVC}\left(T^{*}\right)=\min \left[T V C_{1}\left(T_{1}^{*}\right), T V C_{2}\left(T_{2}^{*}\right),-C I_{p} P R M_{0}\right]$

(f) If $H(2 P-1)+2 C I_{p} P-S I_{e}>0, H(2 P-1)-$ $2 S I_{e} \alpha P R+S I e>0, T_{1}^{*}<\frac{W}{P R}$ and $T_{2}^{*}<\frac{W}{P R}$ then Equations (14),(17) and (23) imply that $T V C(T)$ is decreasing on (0, $\left.T_{1}^{*}\right]$, increasing on $\left[T_{1}^{*}, \frac{W}{P R}\right)$, increasing on $\left[\frac{W}{P R}, \frac{M_{0}}{1-\alpha P R}\right)$ and 
decreasing on $\left[\frac{M_{0}}{1-\alpha P R}, \infty\right)$. Since $\lim _{T \rightarrow \frac{W}{P R}} T V C_{1}(T)>T V C_{2}\left(\frac{W}{P R}\right)$, so we conclude that $T^{*}=T_{1}^{*}, \frac{T \rightarrow \frac{W}{P R}}{P R}$ or $\infty$ (associated with the least cost) $\operatorname{TVC}\left(T^{*}\right)=\min \left[T V C_{1}\left(T_{1}^{*}\right)\right.$, $\left.T V C_{2}\left(\frac{W}{P R}\right),-C I_{p} P R M_{0}\right](\mathbf{C})$ Suppose that $H(2 P-1)+$ $2 C I_{p} P-2 C I_{p} \alpha P^{2} R-S I_{e}>0, H(2 P-1)+2 C I_{p} P-$ $S I_{e}>0$ and

(i) if $H(2 P-1)-2 S I_{e} \alpha P R+S I e \leq 0$ then

(a) If $T_{1}^{*}<\frac{W}{P R}$ and $T_{3}^{*} \geq \frac{M_{0}}{1-\alpha P R}$ then $\operatorname{TVC}\left(T^{*}\right)=\min$ $\left[T V C_{1}\left(T_{1}^{*}\right), T V C_{3}\left(T_{3}^{*}\right)\right]$ and $T^{*}=T_{1}^{*}$ or $T_{3}^{*}$ (associated with the least cost).

(b) If $T_{1}^{*}<\frac{W}{P R}$ and $T_{3}^{*}<\frac{M_{0}}{1-\alpha P R}$ then $\operatorname{TVC}\left(T^{*}\right)=\min$ $\left[T V C_{1}\left(T_{1}^{*}\right), T V C_{3}\left(\frac{M_{0}}{1-\alpha P R}\right)\right]$ and $T^{*}=T_{1}^{*}$ or $\frac{M_{0}}{1-\alpha P R}$ (associated with the least cost).

(c) If $T_{1}^{*} \geq \frac{W}{P R}$ and $T_{3}^{*} \geq \frac{M_{0}}{1-\alpha P R}$ then $\operatorname{TVC}\left(T^{*}\right)=$ $T V C_{3}\left(T_{3}^{*}\right)$ and $T^{*}=T_{3}^{*}$.

(d) If $T_{1}^{*} \geq \frac{W}{P R}$ and $T_{3}^{*}<\frac{M_{0}}{1-\alpha P R}$ then $\operatorname{TVC}\left(T^{*}\right)=$ $T V C_{3}\left(\frac{M_{0}}{1-\alpha P R}\right)$ and $T^{*}=\frac{M_{0}}{1-\alpha P R}$.

(ii) if $H(2 P-1)-2 S I_{e} \alpha P R+S I e>0$ then

(a) If $T_{1}^{*}<\frac{W}{P R}, T_{2}^{*}<\frac{W}{P R}$ and $T_{3}^{*}<\frac{M_{0}}{1-\alpha P R}$ then $\operatorname{TVC}\left(T^{*}\right)=\min \left[T V C_{1}\left(T_{1}^{*}\right), T V C_{2}\left(\frac{W}{P R}\right)\right]$ and $T^{*}=T_{1}^{*}$ or $\frac{W}{P R}$ (associated with the least cost).

(b) If $T_{1}^{*}<\frac{W}{P R}, T_{2}^{*}<\frac{W}{P R}$ and $T_{3}^{*} \geq \frac{M_{0}}{1-\alpha P R}$ then $\operatorname{TVC}\left(T^{*}\right)=\min \left[T V C_{1}\left(T_{1}^{*}\right), T V C_{3}\left(T_{3}^{*}\right)\right]$ and $T^{*}=T_{1}^{*}$ or $T_{3}^{*}$ (associated with the least cost).

(c) If $T_{1}^{*}<\frac{W}{P R}, \frac{W}{P R} \leq T_{2}^{*} \leq \frac{M_{0}}{1-\alpha P R}$ and $T_{3}^{*}<\frac{M_{0}}{1-\alpha P R}$ then $\operatorname{TVC}\left(T^{*}\right)=\min \left[T V C_{1}\left(T_{1}^{*}\right), T V C_{2}\left(T_{2}^{*}\right)\right]$ and $T^{*}=T_{1}^{*}$ or $T_{2}^{*}$ (associated with the least cost).

(d) If $T_{1}^{*}<\frac{W}{P R}, \frac{W}{P R} \leq T_{2}^{*} \leq \frac{M_{0}}{1-\alpha P R}$ and $T_{3}^{*} \geq \frac{M_{0}}{1-\alpha P R}$ then $\operatorname{TVC}\left(T^{*}\right)=\min \left[T V C_{1}\left(T_{1}^{*}\right), T V C_{2}\left(T_{2}^{*}\right), T V C_{3}\left(T_{3}^{*}\right)\right]$ and $T^{*}=T_{1}^{*}$ or $T_{2}^{*}$ or $T_{3}^{*}$ (associated with the least cost).

(e) If $T_{1}^{*}<\frac{W}{P R}, T_{2}^{*}>\frac{M_{0}}{1-\alpha P R}$ and $T_{3}^{*}<\frac{M_{0}}{1-\alpha P R}$ then $\operatorname{TVC}\left(T^{*}\right)=\min \left[T V C_{1}\left(T_{1}^{*}\right), T V C_{3}\left(\frac{M_{0}}{1-\alpha P R}\right)\right]$ and $T^{*}=T_{1}^{*}$ or $\frac{M_{0}}{1-\alpha P R}$ (associated with the least cost).

(f) If $T_{1}^{*}<\frac{W}{P R}, T_{2}^{*}>\frac{M_{0}}{1-\alpha P R}$ and $T_{3}^{*} \geq \frac{M_{0}}{1-\alpha P R}$ then $\operatorname{TVC}\left(T^{*}\right)=\min \left[T V C_{1}\left(T_{1}^{*}\right), T V C_{3}\left(T_{3}^{*}\right)\right]$ and $T^{*}=T_{1}^{*}$ or $T_{3}^{*}$ (associated with the least cost).

(g) If $T_{1}^{*} \geq \frac{W}{P R}, T_{2}^{*}<\frac{W}{P R}$ and $T_{3}^{*}<\frac{M_{0}}{1-\alpha P R}$ then $\operatorname{TVC}\left(T^{*}\right)=$ $T V C_{2}\left(\frac{W}{P R}\right)$ and $T^{*}=\frac{W}{P R}$. (h) If $T_{1}^{*} \geq \frac{W}{P R}, T_{2}^{*}<\frac{W}{P R}$ and $T_{3}^{*} \geq \frac{M_{0}}{1-\alpha P R}$ then $\operatorname{TVC}\left(T^{*}\right)=\min \left[T V C_{2}\left(\frac{W}{P R}\right), T V C_{3}\left(T_{3}^{*}\right)\right]$ and $T^{*}=\frac{W}{P R}$ or $T_{3}^{*}$ (associated with the least cost).

(i) If $T_{1}^{*} \geq \frac{W}{P R}, \frac{W}{P R} \leq T_{2}^{*} \leq \frac{M_{0}}{1-\alpha P R}$ and $T_{3}^{*}<\frac{M_{0}}{1-\alpha P R}$ then $\operatorname{TVC}\left(T^{*}\right)=T V C_{2}\left(T_{2}^{*}\right)$ and $T^{*}=T_{2}^{*}$.

(j) If $T_{1}^{*} \geq \frac{W}{P R}, \frac{W}{P R} \leq T_{2}^{*} \leq \frac{M_{0}}{1-\alpha P R}$ and $T_{3}^{*} \geq \frac{M_{0}}{1-\alpha P R}$ then $\operatorname{TVC}\left(T^{*}\right)=\min \left[T V C_{2}\left(T_{2}^{*}\right), T V C_{3}\left(T_{3}^{*}\right)\right]$ and $T^{*}=T_{2}^{*}$ or $T_{3}^{*}$ (associated with the least cost).

(k) If $T_{1}^{*} \geq \frac{W}{P R}, T_{2}^{*}>\frac{M_{0}}{1-\alpha P R}$ and $T_{3}^{*}<\frac{M_{0}}{1-\alpha P R}$ then $\operatorname{TVC}\left(T^{*}\right)=T V C_{2}\left(\frac{M_{0}}{1-\alpha P R}\right)$ and $T^{*}=\frac{M_{0}}{1-\alpha P R}$.

(l) If $T_{1}^{*} \geq \frac{W}{P R}, T_{2}^{*}>\frac{M_{0}}{1-\alpha P R}$ and $T_{3}^{*} \geq \frac{M_{0}}{1-\alpha P R}$ then $\operatorname{TVC}\left(T^{*}\right)=T V C_{3}\left(T_{3}^{*}\right)$ and $T^{*}=T_{3}^{*}$.

\section{Proof: (C)}

(i)(a) If $H(2 P-1)+2 C I_{p} P-2 C I_{p} \alpha P^{2} R-S I_{e}>0$, $H(2 P-1)+2 C I_{p} P-S I_{e}>0, \quad H(2 P-1)-$ $2 S I_{e} \alpha P R+S I e \leq 0$ and $T_{1}^{*}<\frac{W}{P R}$ and $T_{3}^{*} \geq \frac{M_{0}}{1-\alpha P R}$ then Equations (12) and (17) imply that TVC (T) is decreasing on $\left(0, T_{1}^{*}\right]$, increasing on $\left[T_{1}^{*}, \frac{W}{P R}\right)$, decreasing on $\left[\frac{W}{P R}, T_{3}^{*}\right]$, and increasing on $\left[T_{3}^{*}, \infty\right)$. Hence $\operatorname{TVC}\left(T^{*}\right)=\min$ $\left[T V C_{1}\left(T_{1}^{*}\right), T V C_{3}\left(T_{3}^{*}\right)\right]$ and $T^{*}=T_{1}^{*}$ or $T_{3}^{*}$ (associated with the least cost).

(b) If $H(2 P-1)+2 C I_{p} P-2 C I_{p} \alpha P^{2} R-S I_{e}>0$, $H(2 P-1)+2 C I_{p} P-S I_{e}>0 \quad, \quad H(2 P-1)-$ $2 S I_{e} \alpha P R+S I e \leq 0$ and $T_{1}^{*}<\frac{W}{P R}$ and $T_{3}^{*}<\frac{M_{0}}{1-\alpha P R}$ then Equations (12), (17) and (25) imply that $\operatorname{TVC}(T)$ is decreasing on $\left(0, T_{1}^{*}\right]$, increasing on $\left[T_{1}^{*}, \frac{W}{P R}\right)$, decreasing on $\left[\frac{W}{P R}, \frac{M_{0}}{1-\alpha P R}\right]$, and increasing on $\left[\frac{M_{0}}{1-\alpha P R}, \infty\right)$. Hence $\operatorname{TVC}\left(T^{*}\right)=\min \left[T V C_{1}\left(T_{1}^{*}\right), T V C_{3}\left(\frac{M_{0}}{1-\alpha P R}\right)\right]$ and $T^{*}=T_{1}^{*}$ or $\frac{M_{0}}{1-\alpha P R}$ (associated with the least cost).

(c) If $H(2 P-1)+2 C I_{p} P-2 C I_{p} \alpha P^{2} R-S I_{e}>0$, $H(2 P-1)+2 C I_{p} P-S I_{e}>0, \quad H(2 P-1)-$ $2 S I_{e} \alpha P R+S I e \leq 0, T_{1}^{*} \geq \frac{W}{P R}$ and $T_{3}^{*} \geq \frac{M_{0}}{1-\alpha P R}$ then Equations (12) , (22) and(17) imply that $\operatorname{TVC}(T)$ is decreasing on $\left(0, T_{3}^{*}\right]$ and increasing on $\left[T_{3}^{*}, \infty\right)$. Hence $T V C\left(T^{*}\right)=T V C_{3}\left(T_{3}^{*}\right)$ and $T^{*}=T_{3}^{*}$.

(d) If $H(2 P-1)+2 C I_{p} P-2 C I_{p} \alpha P^{2} R-S I_{e}>0$, $H(2 P-1)+2 C I_{p} P-S I_{e}>0 \quad, \quad H(2 P-1)-$ $2 S I_{e} \alpha P R+S I e \leq 0, T_{1}^{*} \geq \frac{W}{P R}$ and $T_{3}^{*}<\frac{M_{0}}{1-\alpha P R}$ then Equations (12) , (22) and(25) imply that $\operatorname{TVC}(T)$ is decreasing on $\left(0, \frac{M_{0}}{1-\alpha P R}\right]$ and increasing on $\left[\frac{M_{0}}{1-\alpha P R}, \infty\right)$. Hence $\operatorname{TVC}\left(T^{*}\right)=\operatorname{TV} C_{3}\left(\frac{M_{0}}{1-\alpha P R}\right)$ and $T^{*}=\frac{M_{0}}{1-\alpha P R}$. 
(ii)(a) If $H(2 P-1)+2 C I_{p} P-2 C I_{p} \alpha P^{2} R-S I_{e}>0$, $H(2 P-1)+2 C I_{p} P-S I_{e}>0 \quad, \quad H(2 P-1)-$ $2 S I_{e} \alpha P R+S I e>0$ and $T_{1}^{*}<\frac{W}{P R}, T_{2}^{*}<\frac{W}{P R}$ and $T_{3}^{*}<\frac{M_{0}}{1-\alpha P R}$ then Equations (17),(23) and (25) imply that $T V C(T)$ is decreasing on $\left(0, T_{1}^{*}\right]$ and increasing on $\left[T_{1}^{*}, \infty\right)$. Since $\lim _{T \rightarrow \frac{W}{P R}} T V C_{1}(T)>T V C_{2}\left(\frac{W}{P R}\right)$, so we conclude that then $\operatorname{TVC}\left(T^{*}\right)=\min \left[T V C_{1}\left(T_{1}^{*}\right), T V C_{2}\left(\frac{W}{P R}\right)\right]$ and $T^{*}=T_{1}^{*}$ or $\frac{W}{P R}$ (associated with the least cost).

(b) If $H(2 P-1)+2 C I_{p} P-2 C I_{p} \alpha P^{2} R-S I_{e}>0$, $H(2 P-1)+2 C I_{p} P-S I_{e}>0 \quad, \quad H(2 P-1)-$ $2 S I_{e} \alpha P R+S I e>0$ and $T_{1}^{*}<\frac{W}{P R}, T_{2}^{*}<\frac{W}{P R}$ and $T_{3}^{*} \geq$ $\frac{M_{0}}{1-\alpha P R}$ then Equations (17) and (23) imply that $T V C(T)$ is decreasing on $\left(0, T_{1}^{*}\right]$, increasing on $\left[T_{1}^{*}, \frac{M_{0}}{1-\alpha P R}\right]$, decreasing on $\left[\frac{M_{0}}{1-\alpha P R}, T_{3}^{*}\right]$ and increasing on $\left[T_{3}^{*}, \infty\right)$. Hence $\operatorname{TVC}\left(T^{*}\right)=\min \left[T V C_{1}\left(T_{1}^{*}\right), T V C_{3}\left(T_{3}^{*}\right)\right]$ and $T^{*}=$ $T_{1}^{*}$ or $T_{3}^{*}$ (associated with the least cost).

(c) If $H(2 P-1)+2 C I_{p} P-2 C I_{p} \alpha P^{2} R-S I_{e}>0$, $H(2 P-1)+2 C I_{p} P-S I_{e}>0 \quad, \quad H(2 P-1)-$ $2 S I_{e} \alpha P R+S I e>0$ and $T_{1}^{*}<\frac{W}{P R}, \frac{W}{P R} \leq T_{2}^{*} \leq \frac{M_{0}}{1-\alpha P R}$ and $T_{3}^{*}<\frac{M_{0}}{1-\alpha P R}$ then Equations (17) and (25) imply that $T V C(T)$ is decreasing on $\left(0, T_{1}^{*}\right]$, increasing on $\left[T_{1}^{*}, \frac{W}{P R}\right)$, decreasing on $\left[\frac{W}{P R}, T_{2}^{*}\right]$ and increasing on $\left[T_{2}^{*}, \infty\right)$. Hence $\operatorname{TVC}\left(T^{*}\right)=\min \left[T V C_{1}\left(T_{1}^{*}\right), T V C_{2}\left(T_{2}^{*}\right)\right]$ and $T^{*}=T_{1}^{*}$ or $T_{2}^{*}$ (associated with the least cost).

(d) If $H(2 P-1)+2 C I_{p} P-2 C I_{p} \alpha P^{2} R-S I_{e}>0$, $H(2 P-1)+2 C I_{p} P-S I_{e}>0 \quad, \quad H(2 P-1)-$ $2 S I_{e} \alpha P R+S I e>0$ and $T_{1}^{*}<\frac{W}{P R}, \frac{W}{P R} \leq T_{2}^{*} \leq \frac{M_{0}}{1-\alpha P R}$ and $T_{3}^{*} \geq \frac{M_{0}}{1-\alpha P R}$ then Equation (17) implies that $T V C(T)$ is decreasing on $\left(0, T_{1}^{*}\right]$, increasing on $\left[T_{1}^{*}, \frac{W}{P R}\right)$, decreasing on $\left[\frac{W}{P R}, T_{2}^{*}\right]$, increasing on $\left[T_{2}^{*}, \frac{M_{0}}{1-\alpha P R}\right]$, decreasing on $\left[\frac{M_{0}}{1-\alpha P R}, T_{3}^{*}\right]$ and increasing on $\left[T_{3}^{*}, \infty\right)$. Hence $\operatorname{TVC}\left(T^{*}\right)=$ $\min \left[T V C_{1}\left(T_{1}^{*}\right), T V C_{2}\left(T_{2}^{*}\right), T V C_{3}\left(T_{3}^{*}\right)\right]$ and $T^{*}=T_{1}^{*}$ or $T_{2}^{*}$ or $T_{3}^{*}$ (associated with the least cost).

(e) If $H(2 P-1)+2 C I_{p} P-2 C I_{p} \alpha P^{2} R-S I_{e}>0$, $H(2 P-1)+2 C I_{p} P-S I_{e}>0 \quad, \quad H(2 P-1)-$ $2 S I_{e} \alpha P R+S I e>0$ and $T_{1}^{*}\left\langle\frac{W}{P R}, T_{2}^{*}>\frac{M_{0}}{1-\alpha P R}\right.$ and $T_{3}^{*}<$ $\frac{M_{0}}{1-\alpha P R}$ then Equations (17), (24) and (25) imply that $\operatorname{TVC}(T)$ is decreasing on $\left(0, T_{1}^{*}\right]$, increasing on $\left[T_{1}^{*}, \frac{W}{P R}\right)$, decreasing on $\left[\frac{W}{P R}, \frac{M_{0}}{1-\alpha P R}\right]$ and increasing on $\left[\frac{M_{0}}{1-\alpha P R}, \infty\right)$. Hence $\operatorname{TVC}\left(T^{*}\right)=\min \left[T V C_{1}\left(T_{1}^{*}\right), \operatorname{TVC}_{3}\left(\frac{M_{0}}{1-\alpha P R}\right)\right]$ and $T^{*}$ $=T_{1}^{*}$ or $\frac{M_{0}}{1-\alpha P R}$ (associated with the least cost).

(f) If $H(2 P-1)+2 C I_{p} P-2 C I_{p} \alpha P^{2} R-S I_{e}>0$,
$H(2 P-1)+2 C I_{p} P-S I_{e}>0 \quad, \quad H(2 P-1)-$ $2 S I_{e} \alpha P R+S I e>0$ and $T_{1}^{*}\left\langle\frac{W}{P R}, T_{2}^{*}>\frac{M_{0}}{1-\alpha P R}\right.$ and $T_{3}^{*} \geq$ $\frac{M_{0}}{1-\alpha P R}$ then Equations (17)and (24) imply that $T V C(T)$ is decreasing on $\left(0, T_{1}^{*}\right]$, increasing on $\left[T_{1}^{*}, \frac{W}{P R}\right)$, decreasing on $\left[\frac{W}{P R}, T_{3}^{*}\right]$ and increasing on $\left[T_{3}^{*}, \infty\right)$. Hence $\operatorname{TVC}\left(T^{*}\right)=$ $\min \left[T V C_{1}\left(T_{1}^{*}\right), T V C_{3}\left(T_{3}^{*}\right)\right]$ and $T^{*}=T_{1}^{*}$ or $T_{3}^{*}$ (associated with the least cost).

(g) If $H(2 P-1)+2 C I_{p} P-2 C I_{p} \alpha P^{2} R-S I_{e}>0$, $H(2 P-1)+2 C I_{p} P-S I_{e}>0, \quad H(2 P-1)-$ $2 S I_{e} \alpha P R+S I e>0$ and $T_{1}^{*} \geq \frac{W}{P R}, T_{2}^{*}<\frac{W}{P R}$ and $T_{3}^{*}$ $<\frac{M_{0}}{1-\alpha P R}$ then Equations (17), (23) and (25) imply that $\operatorname{TVC}(T)$ is decreasing on $\left(0, \frac{W}{P R}\right)$ and increasing on $\left[\frac{W}{P R}, \infty\right)$. Hence $\operatorname{TVC}\left(T^{*}\right)=T V C_{2}\left(\frac{W}{P R}\right)$ and $T^{*}=\frac{W}{P R}$.

(h) If $H(2 P-1)+2 C I_{p} P-2 C I_{p} \alpha P^{2} R-S I_{e}>0$, $H(2 P-1)+2 C I_{p} P-S I_{e}>0 \quad, \quad H(2 P-1)-$ $2 S I_{e} \alpha P R+S I e>0$ and $T_{1}^{*} \geq \frac{W}{P R}, T_{2}^{*}<\frac{W}{P R}$ and $T_{3}^{*} \geq$ $\frac{M_{0}}{1-\alpha P R}$ then Equations (22), (23)and (17) imply that $\operatorname{TVC}(T)$ is decreasing on $\left(0, \frac{W}{P R}\right)$, increasing on $\left[\frac{W}{P R}\right.$, $\left.\frac{M_{0}}{1-\alpha P R}\right]$, decreasing on $\left[\frac{M_{0}}{1-\alpha P R}, T_{3}^{*}\right]$ and increasing on $\left[T_{3}^{*}\right.$, $\infty)$. Hence $\operatorname{TVC}\left(T^{*}\right)=\min \left[T V C_{2}\left(\frac{W}{P R}\right), T V C_{3}\left(T_{3}^{*}\right)\right]$ and $T^{*}=\frac{W}{P R}$ or $T_{3}^{*}$ (associated with the least cost).

(i) If $H(2 P-1)+2 C I_{p} P-2 C I_{p} \alpha P^{2} R-S I_{e}>0$, $H(2 P-1)+2 C I_{p} P-S I_{e}>0, \quad H(2 P-1)-$ $2 S I_{e} \alpha P R+S I e>0$ and $T_{1}^{*} \geq \frac{W}{P R}, \frac{W}{P R} \leq T_{2}^{*} \leq \frac{M_{0}}{1-\alpha P R}$ and $T_{3}^{*}<\frac{M_{0}}{1-\alpha P R}$ then Equations (22), (17) and (25) imply that $T V C(T)$ is decreasing on $\left(0, T_{2}^{*}\right]$ and increasing on $\left[T_{2}^{*}\right.$, $\infty)$. Hence $\operatorname{TVC}\left(T^{*}\right)=T V C_{2}\left(T_{2}^{*}\right)$ and $T^{*}=T_{2}^{*}$.

(j) If $H(2 P-1)+2 C I_{p} P-2 C I_{p} \alpha P^{2} R-S I_{e}>0$, $H(2 P-1)+2 C I_{p} P-S I_{e}>0 \quad, \quad H(2 P-1)-$ $2 S I_{e} \alpha P R+S I e>0$ and $T_{1}^{*} \geq \frac{W}{P R}, \frac{W}{P R} \leq T_{2}^{*} \leq \frac{M_{0}}{1-\alpha P R}$ and $T_{3}^{*} \geq \frac{M_{0}}{1-\alpha P R}$ then Equations (22)and (17) imply that $\operatorname{TVC}(T)$ is decreasing on $\left(0, T_{2}^{*}\right]$, increasing on $\left[T_{2}^{*}\right.$, $\left.\frac{M_{0}}{1-\alpha P R}\right]$, decreasing on $\left[\frac{M_{0}}{1-\alpha P R}, T_{3}^{*}\right]$ and increasing on $\left[T_{3}^{*}\right.$, $\infty)$. Hence $\operatorname{TVC}\left(T^{*}\right)=\min \left[T V C_{2}\left(T_{2}^{*}\right), T V C_{3}\left(T_{3}^{*}\right)\right]$ and $T^{*}$ $=T_{2}^{*}$ or $T_{3}^{*}$ (associated with the least cost).

(k) If $H(2 P-1)+2 C I_{p} P-2 C I_{p} \alpha P^{2} R-S I_{e}>0$, $H(2 P-1)+2 C I_{p} P-S I_{e}>0 \quad, \quad H(2 P-1)-$ $2 S I_{e} \alpha P R+S I e>0$ and $T_{1}^{*} \geq \frac{W}{P R}, T_{2}^{*}>\frac{M_{0}}{1-\alpha P R}$ and $T_{3}^{*}<$ $\frac{M_{0}}{1-\alpha P R}$ then Equations (22), (24)and (25) imply that $\operatorname{TVC}(T)$ is decreasing on $\left(0, \frac{M_{0}}{1-\alpha P R}\right]$ and increasing on $\left[\frac{M_{0}}{1-\alpha P R}, \infty\right)$. Hence $\operatorname{TVC}\left(T^{*}\right)=T V C_{2}\left(\frac{M_{0}}{1-\alpha P R}\right)$ and $T^{*}=$ $\frac{M_{0}}{1-\alpha P R}$. 
(l) If $H(2 P-1)+2 C I_{p} P-2 C I_{p} \alpha P^{2} R-S I_{e}>0$, $H(2 P-1)+2 C I_{p} P-S I_{e}>0 \quad, \quad H(2 P-1)-$ $2 S I_{e} \alpha P R+S I e>0$ and $T_{1}^{*} \geq \frac{W}{P R}, T_{2}^{*}>\frac{M_{0}}{1-\alpha P R}$ and $T_{3}^{*} \geq$ $\frac{M_{0}}{1-\alpha P R}$ then Equations (22), (24)and (17) imply that $\operatorname{TVC}(T)$ is decreasing on $\left(0, T_{3}^{*}\right]$ and increasing on $\left[T_{3}^{*}\right.$, $\infty)$. Hence $\operatorname{TVC}\left(T^{*}\right)=T V C_{3}\left(T_{3}^{*}\right)$ and $T^{*}=T_{3}^{*}$.

\section{Decision Rule of the Optimal Cycle Time When $\frac{W}{P R}>M=M_{0}+$ $\alpha P R T$.}

In this case Equations (10) and (14) yield

$T_{1}^{*} \geq \frac{W}{P R}$ implies $T V C_{1}^{\prime}\left(\frac{W}{P R}\right) \leq 0$ and hence $T V C_{1}(T)$ is decreasing on $\left(0, \frac{W}{P R}\right)$

$T_{3}^{*}<\frac{W}{P R}$ implies $T V C_{3}^{\prime}\left(\frac{W}{P R}\right)>0$ and hence $T V C_{3}(T)$ is

$$
\text { increasing on }\left[\frac{W}{P R}, \infty\right)
$$

Furthermore, the result follows.

\section{Theorem 3.}

(A) Suppose that $H(2 P-1)+2 C I_{p} P-$ $2 C I_{p} \alpha P^{2} R-S I_{e}<0$ then $T^{*}=\infty$ and $\operatorname{TVC}\left(T^{*}\right)=-\infty$ ie., the retailer will try to continue his cycle as much as possible.

Proof: See Theorem 2-(A)

(B) Suppose that $H(2 P-1)+2 C I_{p} P-$ $2 C I_{p} \alpha P^{2} R-S I_{e}=0$ then

(i) If $H(2 P-1)+2 C I_{p} P-S I_{e}=0$ then $T^{*}=\infty$ and $\operatorname{TVC}\left(T^{*}\right)=-C I_{p} P R M_{0}$.

(ii) If $H(2 P-1)+2 C I_{p} P-S I_{e}>0$ then

(a) If $T_{1}^{*} \geq \frac{W}{P R}$ then $T^{*}=\infty$ and $\operatorname{TVC}\left(T^{*}\right)$ $=-C I_{p} P R M_{0}$.

(b) If $T_{1}^{*}<\frac{W}{P R}$ then $T^{*}=T_{1}^{*}$ or $\infty$ and $\operatorname{TVC}\left(T^{*}\right)=$ $\min \left[T V C_{1}\left(T_{1}^{*}\right),-C I_{p} P R M_{0}\right]$.

Proof: (i) If $H(2 P-1)+2 C I_{p} P-2 C I_{p} \alpha P^{2} R-S I_{e}$ $=0$ and $H(2 P-1)+2 C I_{p} P-S I_{e}=0$ then Equation (10) and (14) imply that $\operatorname{TVC}(T)$ is decreasing on $(0, \infty)$. Again $\lim _{T \rightarrow 0^{+}} T V C(T)=\infty$ and $\lim _{T \rightarrow \infty} T V C(T)=-C I_{p} P R M_{0}$. Consequently $T^{*}=\infty$ and $\operatorname{TVC}\left(T^{*}\right)=-C I_{p} P R M_{0}$.

(ii)(a) If $H(2 P-1)+2 C I_{p} P-2 C I_{p} \alpha P^{2} R-S I_{e}=0$, $H(2 P-1)+2 C I_{P} P-S I_{e}=0$ and $T_{1}^{*} \geq \frac{W}{P R}$ then
Equation (14) and (26) imply that $T V C(T)$ is decreasing on $(0, \infty)$. Again $\lim _{T \rightarrow 0^{+}} \operatorname{TV} C(T)=\infty$ and $\lim _{T \rightarrow \infty} \operatorname{TVC}(T)=$ $-C I_{p} P R M_{0}$. Consequently $T^{*}=\infty$ and $\operatorname{TVC}\left(T^{*}\right)$ $=-C I_{p} P R M_{0}$.

(b) If $H(2 P-1)+2 C I_{p} P-2 C I_{p} \alpha P^{2} R-S I_{e}=0$, $H(2 P-1)+2 C I_{p} P-S I_{e}=0$ and $T_{1}^{*}<\frac{W}{P R}$ then Equation (14) and (17) imply that $T V C(T)$ is decreasing on $\left(0, T_{1}^{*}\right]$ and increasing on $\left[T_{1}^{*}, \frac{W}{P R}\right)$ and decreasing on $\left[\frac{W}{P R}, \infty\right)$. Consequently $T^{*}=T_{1}^{*}$ or $\infty$ (linked with the smallest cost) and $\operatorname{TVC}\left(T^{*}\right)=\min \left[T V C_{1}\left(T_{1}^{*}\right)\right.$, $\left.-C I_{p} P R M_{0}\right]$.

(C) Suppose that $H(2 P-1)+2 C I_{p} P-$ $2 C I_{p} \alpha P^{2} R-S I_{e}>0$ then clearly $H(2 P-1)+$ $2 C I_{p} P-S I_{e}>0$ and

(i) If $T_{1}^{*}<\frac{W}{P R}$ and $T_{3}^{*} \geq \frac{W}{P R}$ then $T^{*}=T_{1}^{*}$ or $T_{3}^{*}$ (linked with the smallest cost) and $\operatorname{TVC}\left(T^{*}\right)=\min \left[T V C_{1}\left(T_{1}^{*}\right)\right.$, $\left.T V C_{3}\left(T_{3}^{*}\right)\right]$.

(ii) If $T_{1}^{*} \geq \frac{W}{P R}$ and $T_{3}^{*} \geq \frac{W}{P R}$ then $T^{*}=T_{3}^{*}$ and $\operatorname{TVC}\left(T^{*}\right)=T V C_{3}\left(T_{3}^{*}\right)$.

(iii) If $T_{1}^{*}<\frac{W}{P R}$ and $T_{3}^{*}<\frac{W}{P R}$ then $T^{*}=T_{1}^{*}$ and $\operatorname{TVC}\left(T^{*}\right)=T V C_{1}\left(T_{1}^{*}\right)$.

(iv) If $T_{1}^{*} \geq \frac{W}{P R}$ and $T_{3}^{*}<\frac{W}{P R}$ then $T^{*}=\frac{W}{P R}$ and $\operatorname{TVC}\left(T^{*}\right)=T V C_{3}\left(\frac{W}{P R}\right)$.

\section{Proof:}

(i) If $T_{1}^{*}<\frac{W}{P R}$ and $T_{3}^{*} \geq \frac{W}{P R}$ then Equation (17) implies that $\operatorname{TVC}(T)$ is decreasing on $\left(0, T_{1}^{*}\right]$, increasing on $\left[T_{1}^{*}\right.$, $\left.\frac{W}{P R}\right)$, decreasing on $\left[\frac{W}{P R}, T_{3}^{*}\right]$ and increasing on $\left[T_{3}^{*}, \infty\right)$. Consequently $T^{*}=T_{1}^{*}$ or $T_{3}^{*}$ (linked with the smallest cost) and $\operatorname{TVC}\left(T^{*}\right)=\min \left[T V C_{1}\left(T_{1}^{*}\right), T V C_{3}\left(T_{3}^{*}\right)\right]$.

(ii) If $T_{1}^{*} \geq \frac{W}{P R}$ and $T_{3}^{*} \geq \frac{W}{P R}$ then Equations (26) and (17) imply that $\operatorname{TVC}(T)$ is decreasing on $\left(0, T_{3}^{*}\right]$ and increasing on $\left[T_{3}^{*}, \infty\right)$. So $T^{*}=T_{3}^{*}$ and $\operatorname{TVC}\left(T^{*}\right)$ $=T V C_{3}\left(T_{3}^{*}\right)$.

(iii) If $T_{1}^{*}<\frac{W}{P R}$ and $T_{3}^{*}<\frac{W}{P R}$ then Equations (17) and (25) imply that $\operatorname{TVC}(T)$ is decreasing on $\left(0, T_{1}^{*}\right]$ and increasing on $\left[T_{1}^{*}, \infty\right)$. So $T^{*}=T_{1}^{*}$ and $\operatorname{TVC}\left(T^{*}\right)=$ $T V C_{1}\left(T_{1}^{*}\right)$.

(iv) If $T_{1}^{*} \geq \frac{W}{P R}$ and $T_{3}^{*}<\frac{W}{P R}$ then Equations (26) and (17) imply that $\operatorname{TVC}(T)$ is decreasing on $\left(0, \frac{W}{P R}\right)$ and increasing on $\left[\frac{W}{P R}, \infty\right)$. So $T^{*}=\frac{W}{P R}$ and $\operatorname{TVC}\left(T^{*}\right)=$ $T V C_{3}\left(\frac{W}{P R}\right)$. 


\section{Algorithm}

Step 1. If $\alpha P R \geq 1$, go to step 5 .

Step 2. Find $T^{*}$ from Theorem 2.

Step 3. If $\frac{W}{P R} \leq M_{0}+\alpha P R T^{*}$, then $T_{0}^{*}=T^{*}$.

Step 4. Go to step 7.

Step 5. Find $T^{*}$ from Theorem 1 .

Step 6. If $\frac{W}{P R} \leq M_{0}+\alpha P R T^{*}$, then $T_{0}^{*}=T^{*}$.

Step 7. Find $T^{*}$ from Theorem 3 .

Step 8. If $\frac{W}{P R}>M_{0}+\alpha P R T^{*}$, then $T_{00}^{*}=T^{*}$.

Step 9. If only $T_{0}^{*}$ exists and $T_{00}^{*}$ does not exist, then $T_{0}^{*}$ is the optimal cycle time.

Step 10 . If only $T_{00}^{*}$ exists and $T_{0}^{*}$ does not exist, then $T_{00}^{*}$ is the optimal cycle time.

Step 11. If both $T_{0}^{*}$ and $T_{00}^{*}$ exist, then calculate $\operatorname{TVC}\left(T_{0}^{*}\right)$ and $T V C\left(T_{00}^{*}\right)$.

Step 12. If $T V C\left(T_{0}^{*}\right) \geq \operatorname{TVC}\left(T_{00}^{*}\right)$, then optimum cycle time is $T_{00}^{*}$, otherwise $T_{0}^{*}$ is the optimal cycle time.

\section{Numerical Example}

Let us study inventory structure with the subsequent parameters in suitable units.

(i) Let the probability density of demand $x \mathrm{~kg}$ of the item throughout period $T$ month be uniform in $a(T)=$ $10 T \leq x \leq b(T)=60 T \quad$ i.e., $\quad f(x \mid T)=$ $\left\{\begin{array}{cc}\frac{1}{a(T)-b(T)}, a(T) \leq x \leq b(T) \\ 0, & \text { otherwise }\end{array}\right.$. Therefore we get $\mu(T)=$ $35 T, P=1.7, R=\frac{\mu(T)}{T}=35$. Other parameters are $A=\$ 50$ per cycle, $H=\$ 0.5$ per kg per month, $C=$ $\$ 10$ per $\mathrm{kg}, S=\$ 12$ per $\mathrm{kg}, \alpha=0.5, M_{0}=2$ month, $I_{e}=\$ 0.025$ per $\$$ per month, $I_{p}=\$ 0.05$ per $\$$ per month, $W=30$ (here $R \geq 1$ ). Using Theorem 1 , we get $T^{*}=\infty, \operatorname{TVC}\left(T^{*}\right)=-\infty$ and $\frac{W}{P R} \leq M_{0}+\alpha P R T^{*}$ is satisfied. Again using Theorem 3, we get $T^{*}=\infty$, $V C\left(T^{*}\right)=-\infty$, and $\frac{W}{P R}>M_{0}+\alpha P R T^{*}$, is not satisfied. Hence optimal cycle time is infinity and optimal cost is minus infinity (i.e., the retailer will try to continue production cycle as much as possible).

(ii) Let the probability density of demand $x \mathrm{~kg}$ of the item throughout period $T$ month be uniform in $a(T)=$ $0 \leq x \leq b(T)=20 T \quad$ i.e., $\quad f(x \mid T)=$ $\left\{\begin{array}{cc}\frac{1}{a(T)-b(T)}, a(T) \leq x \leq b(T) \\ 0, \quad \text { otherwise }\end{array}\right.$. Therefore we get $\mu(T)=$
10T, $P=2, R=\frac{\mu(T)}{T}=10$. Other parameters are $A=$ $\$ 50$ per cycle, $H=\$ 0.5$ per $\mathrm{kg}$ per month, $C=\$ 10$ per kg, $S=\$ 12$ per kg, $\alpha=0.5, M_{0}=2$ month, $I_{e}=$ $\$ 0.005$ per $\$$ per month, $I_{p}=\$ 0.05$ per $\$$ per month, $W=30$ (here $R \geq 1$ ). Using Theorem 1 , we get $T^{*}=$ 5.2705, $\operatorname{TVC}\left(T^{*}\right)=17.77$ and $\frac{W}{P R} \leq M_{0}+\alpha P R T^{*}$ is satisfied. Again using Theorem 3, we get $T^{*}=\infty$, $\operatorname{TVC}\left(T^{*}\right)=-\infty$, and $\frac{W}{P R}>M_{0}+\alpha P R T^{*}$, is not satisfied. Hence optimal cycle time is 5.2705 month and optimal cost is $\$ 17.77$.

(iii) Let the probability density of demand $x \mathrm{~kg}$ of the item throughout period $T$ month be normal with parameters mean $9 T$ and standard deviation $3 T$ in $a(T)=0 \leq x \leq b(T)=18 T \quad$ i.e., $\quad f(x \mid T)=$ $\left\{\begin{array}{cc}\frac{1}{\sqrt{2 \pi} \sigma(T)} e^{-\frac{(x-\mu(T))^{2}}{2 \sigma(T)^{2}}}, a(T) \leq x \leq b(T) \\ 0, & \text { otherwise }\end{array}\right.$. Therefore we get $\mu(T)=9 T, P=2, R=\frac{\mu(T)}{T}=9$. Other parameters are $A=\$ 50$ per cycle, $H=\$ 0.5$ per kg per month, $C=$ $\$ 10$ per kg, $S=\$ 12$ per kg, $\alpha=0.5, M_{0}=2$ month, $I_{e}=\$ 0.005$ per $\$$ per month, $I_{p}=\$ 0.05$ per $\$$ per month, $W=30$ (here $R<1$ ). Using Theorem 2, we get $T^{*}=2.9695, \operatorname{TVC}\left(T^{*}\right)=28.6548$ and $\frac{W}{P R} \leq M_{0}+$ $\alpha P R T^{*}$ is satisfied. Again using Theorem 3, we get $T^{*}=$ 2.8172, $C\left(T^{*}\right)=17.4965$, and $\frac{W}{P R}>M_{0}+\alpha P R T^{*}$, is not satisfied. Hence optimal cycle time is 2.9695 month and optimal cost is $\$ 28.6548$.

\section{Sensitivity Analysis}

At this time, we study two instances and debate the sensitivity investigation of all the parameters in each case.

(I) In the first problem, let the probability density demand $x \mathrm{~kg}$ of the item throughout period $T$ month be uniform in $a(T)=0 \leq x \leq b(T)=18 T$ i.e., $f(x \mid T)=\left\{\begin{array}{cc}\frac{1}{a(T)-b(T)}, a(T) \leq x \leq b(T) \\ 0, & \text { otherwise }\end{array}\right.$. Therefore we get $\mu(T)=9 T, P=2, R=\frac{\mu(T)}{T}=9$. Other parameters are $A=\$ 50$ per cycle, $H=\$ 0.6$ per $\mathrm{kg}$ per month, $C=\$ 10$ per $\mathrm{kg}, S=\$ 13$ per $\mathrm{kg}, \alpha=0.4, M_{0}=2$ month, $I_{e}=\$ 0.004$ per $\$$ per month, $I_{p}=\$ 0.05$ per $\$$ per month, $W=30$. Solving the problem, we obtain, optimal cycle time is 3.1735 month and optimal cost is $\$ 30.5739$.

(II) whereas in the second problem, the probability density of demand $x \mathrm{~kg}$ of the item throughout period $T$ month be normal with parameters mean $10 T$ and standard deviation $0.85 T$ in $a(T)=7.5 T \leq x \leq$ $b(T)=12.5 T \quad$ i.e., $\quad f(x \mid T)=$ 
$\left\{\begin{array}{cc}\frac{1}{\sqrt{2 \pi} \sigma(T)} e^{-\frac{(x-\mu(T))^{2}}{2 \sigma(T)^{2}}}, a(T) \leq x \leq b(T) & \text {. Therefore we get } \\ 0, & \text { otherwise }\end{array}\right.$

$\mu(T)=10 T, P=1.25, R=\frac{\mu(T)}{T}=10$. parameters are $A=\$ 50$ per cycle, $H=\$ 1.5$ per $\mathrm{kg}$ per month, $C=\$ 10$ per $\mathrm{kg}, S=\$ 13$ per $\mathrm{kg}, \alpha=$ $0.05, M_{0}=2$ month, $I_{e}=\$ 0.025$ per $\$$ per month, $I_{p}=\$ 0.05$ per $\$$ per month, $W=35$. The optimal cycle time is 1.7747 month and optimal cost is $\$ 56.3471$.

Table 1. Sensitivity analysis of different parameters

\begin{tabular}{|c|c|c|c|c|c|}
\hline \multirow{2}{*}{ Parameter } & \multirow{2}{*}{ Change } & \multicolumn{2}{|c|}{ Example (I) } & \multicolumn{2}{|c|}{ Example (II) } \\
\hline & & Cycle time & Total Cost & Cycle time & Total Cost \\
\hline \multirow{4}{*}{$\boldsymbol{A}$} & $-20 \%$ & $2.8385(-10.6 \%)$ & $27.2474(-10.9 \%)$ & $1.5873(-10.6 \%)$ & $50.3984(-10.6 \%)$ \\
\hline & $-10 \%$ & $3.0107(-05.1 \%)$ & $28.9570(-05.3 \%)$ & $1.6836(-05.1 \%)$ & $53.4555(-0.51 \%)$ \\
\hline & $+10 \%$ & $3.3284(+04.9 \%)$ & $32.1119(+05.0 \%)$ & $1.8613(+04.8 \%)$ & $59.0973(+04.9 \%$ \\
\hline & $+20 \%$ & $3.4765(+09.5 \%)$ & $33.5814(+09.8 \%)$ & $1.9441(+09.5 \%)$ & $61.7251(+09.5 \%)$ \\
\hline \multirow{4}{*}{$\boldsymbol{H}$} & $-20 \%$ & $3.8665(+20.0 \%)$ & $24.9267(-18.5 \%)$ & $1.9157(+07.9 \%)$ & $52.2015(-07.4 \%)$ \\
\hline & $-10 \%$ & $3.4692(+09.3 \%)$ & $27.8889(-08.8 \%)$ & $1.8411(+03.7 \%)$ & $54.3139(-03.6 \%)$ \\
\hline & $+10 \%$ & $2.9426(-07.3 \%)$ & $33.0475(+08.1 \%)$ & $1.7149(-03.4 \%)$ & $58.3095(+03.5 \%)$ \\
\hline & $+20 \%$ & $2.7556(-13.2 \%)$ & $35.3528(+15.6 \%)$ & $1.6609(-06.4 \%)$ & $60.2079(+06.9 \%)$ \\
\hline \multirow{4}{*}{$\boldsymbol{R}$} & $-20 \%$ & $3.3293(+04.9 \%)$ & $29.2866(-04.2 \%)$ & $1.9841(+11.8 \%)$ & $50.3984(-10.6 \%)$ \\
\hline & $-10 \%$ & $3.2372(+02.0 \%)$ & $30.0484(-01.7 \%)$ & $1.8707(+05.4 \%)$ & $53.4555(-05.1 \%)$ \\
\hline & $+10 \%$ & $3.1341(-01.2 \%)$ & $30.87770(+01.0 \%)$ & $1.6927(-04.6 \%)$ & $59.0973(+04.9 \%)$ \\
\hline & $+20 \%$ & $3.1163(-01.8 \%)$ & $30.9659(+01.3 \%)$ & $1.6200(-08.7 \%)$ & $61.7251(+09.5 \%)$ \\
\hline \multirow{4}{*}{$\alpha$} & $-20 \%$ & $2.9779(-06.2 \%)$ & $32.6447(+06.8 \%)$ & $1.7747(+00.0 \%)$ & $56.3471(+00.0 \%)$ \\
\hline & $-10 \%$ & $3.0710(-03.2 \%)$ & $31.6258(+03.8 \%)$ & $1.7747(+00.0 \%)$ & $56.3471(+00.0 \%)$ \\
\hline & $+10 \%$ & $3.2871(+03.6 \%)$ & $29.4858(-03.5 \%)$ & $1.7747(+00.0 \%)$ & $56.3471(+00.0 \%)$ \\
\hline & $+20 \%$ & $3.4137(+07.6 \%)$ & $28.3572(-07.3 \%)$ & $1.7747(+00.0 \%)$ & $56.3471(+00.0 \%)$ \\
\hline \multirow{4}{*}{$\boldsymbol{P}$} & $-20 \%$ & $3.7914(+19.5 \%)$ & $254394(-16.8 \%)$ & $2.1442(+20.9 \%)$ & $46.6368(-17.2 \%)$ \\
\hline & $-10 \%$ & $3.4415(08.4 \%)$ & $28.1203(-08.0 \%)$ & $1.9334(+08.9 \%)$ & $51.7204(-08.2 \%)$ \\
\hline & $+10 \%$ & $2.9598(-06.7 \%)$ & $32.8499(+07.4 \%)$ & $1.6495(-07.1 \%)$ & $60.6217(+07.6 \%)$ \\
\hline & $+20 \%$ & $2.7841(-12.3 \%)$ & $34.9819(+14.4 \%)$ & $1.5476(-12.8 \%)$ & $64.6142(+14.7 \%)$ \\
\hline \multirow{4}{*}{$I_{e}$} & $-20 \%$ & $2.9903(-05.8 \%)$ & $32.6922(+06.9 \%)$ & $1.7568(-01.8 \%)$ & $56.9201(+01.0 \%)$ \\
\hline & $-10 \%$ & $3.0778(-03.8 \%)$ & $31.6474(+03.5 \%)$ & $1.7656(-00.5 \%)$ & $56.6347(+00.5 \%)$ \\
\hline & $+10 \%$ & $3.2788(+03.3 \%)$ & $29.4690(-03.6 \%)$ & $1.7838(+00.5 \%)$ & $56.0580(-00.5 \%)$ \\
\hline & $+20 \%$ & $3.3952(+06.9 \%)$ & $28.3294(-07.3 \%)$ & $1.7931(+01.0 \%)$ & $55.7673(-01.0 \%)$ \\
\hline \multirow{4}{*}{$I_{p}$} & $-20 \%$ & $3.1735(+00.0 \%)$ & $30.5739(+00.0 \%)$ & $1.8490(+04.2 \%)$ & $54.0833(-04.0 \%)$ \\
\hline & $-10 \%$ & $3.1735(+00.0 \%)$ & $30.5739(+00.0 \%)$ & $1.8107(+02.0 \%)$ & $55.2268(-02.0 \%)$ \\
\hline & $+10 \%$ & $3.1735(+00.0 \%)$ & $30.5739(+00.0 \%)$ & $1.7407(-01.9 \%)$ & $57.4456(+01.9 \%)$ \\
\hline & $+20 \%$ & $3.1735(+00.0 \%)$ & $30.5739(+00.0 \%)$ & $1.7087(-03.7 \%)$ & $58.5234(+03.9 \%)$ \\
\hline \multirow{4}{*}{$\boldsymbol{C}$} & $-20 \%$ & $3.1735(+00.0 \%)$ & $30.5739(00.0 \%)$ & $1.8490(+04.2 \%)$ & $54.0832(-04.0 \%)$ \\
\hline & $-10 \%$ & $3.1735(+00.0 \%)$ & $30.5739(00.0 \%)$ & $1.8107(+02.0 \%)$ & $55.2268(-2.0 \%)$ \\
\hline & $+10 \%$ & $3.1735(+00.0 \%)$ & $30.5739(00.0 \%)$ & $1.7407(-01.9 \%)$ & $57.4456(+01.9 \%)$ \\
\hline & $+20 \%$ & $3.1735(+00.0 \%)$ & $30.5739(00.0 \%)$ & $1.7087(-03.7 \%)$ & $58.5235(+03.9 \%)$ \\
\hline \multirow{4}{*}{$\boldsymbol{S}$} & $-20 \%$ & $2.9903(-05.8 \%)$ & $32.6922(+06.9 \%)$ & $1.7568(-01.8 \%)$ & $56.9209(+01.0 \%)$ \\
\hline & $-10 \%$ & $3.0078(-03.0 \%)$ & $31.6474(+03.5 \%)$ & $1.7656(-00.5 \%)$ & $56.6347(+00.5 \%)$ \\
\hline & $+10 \%$ & $3.2788(+03.3 \%)$ & $29.4690(-03.6 \%)$ & $1.7838(+00.5 \%)$ & $56.0585(-00.5 \%)$ \\
\hline & $+20 \%$ & $3.3952(+06.9 \%)$ & $28.3294(-07.3 \%)$ & $1.7931(+01.0 \%)$ & $55.7673(-01.0 \%)$ \\
\hline
\end{tabular}


Table 1 continued

\begin{tabular}{|c|c|c|c|c|c|}
\hline \multirow{5}{*}{} & $-100 \%$ & $3.1735(+00.0 \%)$ & $30.5739(00.0 \%)$ & $1.7747(00.0 \%)$ & $56.3471(00.0 \%)$ \\
\cline { 2 - 6 } & $-50 \%$ & $3.1735(+00.0 \%)$ & $30.5739(00.0 \%)$ & $1.7747(00.0 \%)$ & $56.3471(00.0 \%)$ \\
\cline { 2 - 6 } & $-20 \%$ & $3.1735(+00.0 \%)$ & $30.5739(00.0 \%)$ & $1.7747(00.0 \%)$ & $56.3471(00.0 \%)$ \\
\cline { 2 - 6 } & $-10 \%$ & $3.1735(+00.0 \%)$ & $30.5739(00.0 \%)$ & $1.7747(00.0 \%)$ & $56.3471(00.0 \%)$ \\
\cline { 2 - 6 } & $+10 \%$ & $3.1735(+00.0 \%)$ & $30.5739(00.0 \%)$ & $1.7747(00.0 \%)$ & $56.3471(00.0 \%)$ \\
\cline { 2 - 6 } & $+20 \%$ & $3.1735(+00.0 \%)$ & $30.5739(00.0 \%)$ & $1.7747(00.0 \%)$ & $56.3471(00.0 \%)$ \\
\cline { 2 - 6 } & $+50 \%$ & $3.1735(+00.0 \%)$ & $30.5739(00.0 \%)$ & $1.7747(00.0 \%)$ & $56.3471(00.0 \%)$ \\
\hline \multirow{5}{*}{$\boldsymbol{N}$} & $+100 \%$ & $1.7218(-45 \%)$ & $58.079250(89.9 \%)$ & $1.7747(00.0 \%)$ & $56.3471(00.0 \%)$ \\
\cline { 2 - 6 } & $-75 \%$ & $3.1735(+00.0 \%)$ & $30.5739(00.0 \%)$ & $2.0439(+15.2 \%)$ & $45.8009(-18.7 \%)$ \\
\cline { 2 - 6 } & $-50 \%$ & $3.1735(+00.0 \%)$ & $30.5739(00.0 \%)$ & $1.7747(00.0 \%)$ & $56.3471(00.0 \%)$ \\
\cline { 2 - 6 } & $-10 \%$ & $3.1735(+00.0 \%)$ & $30.5739(00.0 \%)$ & $1.7747(00.0 \%)$ & $56.3471(00.0 \%)$ \\
\cline { 2 - 6 } & $+10 \%$ & $3.1735(+00.0 \%)$ & $30.5739(00.0 \%)$ & $1.7747(00.0 \%)$ & $56.3471(00.0 \%)$ \\
\cline { 2 - 6 } & $+20 \%$ & $3.1735(+00.0 \%)$ & $30.5739(00.0 \%)$ & $1.7747(00.0 \%)$ & $56.3471(00.0 \%)$ \\
\cline { 2 - 6 } & $+50 \%$ & $3.1735(+00.0 \%)$ & $30.5739(00.0 \%)$ & $1.7747(00.0 \%)$ & $56.3471(00.0 \%)$ \\
\cline { 2 - 6 } & $+75 \%$ & $3.1735(+00.0 \%)$ & $30.5739(00.0 \%)$ & $1.7747(00.0 \%)$ & $56.3471(00.0 \%)$ \\
\hline
\end{tabular}

The Table-1 represents the sensitivity of decision variable 'cycle time' and total cost to changes in each of the 11 parameters in both the problems. Here we observe that cycle time and total cost are moderately sensitive to changes in the parameters $A, H, R, P, I_{e}$ and, that is, even a small change in the values of those parameters make significant change in the decision parameters and total cost. Here we also note that in problem (I) change in $C$ or $I_{p}$ does not change the values of cycle time and total cost whereas in problem (II) cycle time and total cost undergo significant changes with the changes in the values of $C$ or $I_{p}$. Again, in problem (II) a change in $\alpha$ does not change the values of cycle time and total cost whereas in problem (I) cycle time and total cost undergo significant changes when $\alpha$ is changed. From the sensitivity of problem (I) and problem (II), we can conclude that the sensitivity of the parameters $C, I_{p}$ and $\alpha$ are entirely dependent of parameter values of distinct problems. In general, we can conclude about the sensitivity of these parameters. However, cycle time and total cost are not sensitive at all to changes in $W$ and $M_{0}$. But if we make outstanding variations in their values the result may experience noticeable changes. Finally, the eff ects of anew defined parameters can be profoundly detected from the overhead table. It is noted that as $\alpha$ increases, the total cost rises whereas cycle time (not strictly) in case of same result will hold in strict sense. This indicates just how variable trade credit is significant in optimal consequence.

\section{Special Case}

When $P=1$ and $\alpha=0(H=h, S=s, C=c)$. Let $M=M_{0}, D=R$ and

$$
\begin{gathered}
T V C_{4}(T)=\frac{D T h}{2}+c I_{p} D T-\frac{D T s I_{e}}{2} \\
T V C_{5}(T)=\frac{D T h}{2}-D s I_{e}\left[M-\frac{T}{2}\right] \\
T V C_{6}(T)=\frac{D T h}{2}+c I_{p} D(T-M)-\frac{D T s I_{e}}{2} \\
T_{4}^{*}=T_{6}^{*}=\sqrt{\frac{2 A}{D\left(h+2 c I_{p}-s I_{e}\right)}} \\
T_{5}^{*}=\sqrt{\frac{2 A}{D\left(h+s I_{e}\right)}}
\end{gathered}
$$

Then Equations (28), (29), (30), (31), and (32) will be consistent with Equations (2), (3), (4), (12) and (13) in Chung et al.'s model [6] respectively. Again $H(2 P-$ 1) $+2 C I_{p} P-2 C I_{p} \alpha P^{2} R-S I_{e}=H(2 P-1)+$ $2 C I_{p} P-S I_{e}, H(2 P-1)-2 S I_{e} \alpha P R+S I e=\mathrm{h}+\mathrm{sI}_{e}>0$ and $\alpha P R=0<1$. So Theorem 1, Theorem $2(B(i), B(i i i), B(i v), C(i)) \quad$ and Theorem $3(B(i i), C(i), C(i v))$ will not be required. However, other theorems will be consistent with Chung et al.'s [6] model. Thus Chung et al.'s [6] model is a special case of this model.

\section{Conclusions}

This paper deals with a probabilistic economic order quantity inventory model under condition of permissible delay in payments to take the order quantity into account. To reflect realistic commercial circumstances, it is supposed that the trade credit period is not only allied to the order quantity but also varies with the ordering quantity. If $<W$, the delay in payments is not allowed. Else, a flexible trade credit period $M=M_{0}+\alpha Q$ is permitted. It is also supposed that demand rate follows a probability density function. Under these conventions, the model is 
settled. It is shown that, if $\geq \frac{W}{P R}$, one can swiftly determine the optimal ordering quantity by using Theorem 3 . Otherwise, if $<\frac{W}{P R}$, then the optimal ordering strategy can be found from Theorem 1 and Theorem 2. We develop an algorithm, which will support one to determine the optimal $T^{*}$ efficiently. Numerical examples are provided for illustration. To check the fluctuations in the decision variables for changes in diff erent parameters, a sensitivity scrutiny is also carried out. Lastly, we have shown that Chung et al.'s model [6] is a special case of our model.

\section{REFERENCES}

[1] Aggarwal SP, Jaggi CK. Ordering policies of deteriorating items under permissible delay in payments. Journal of the Operation Research Society. Vol. 46, 652-662, 1995

[2] Chang CT, Ouyang LY, Teng JT. An EOQ model for deteriorating items under supplier credits linked to ordering quantity. Applied Mathematical Modelling. Vol. 27, No.12, 983-996, 2003

[3] Chang HC, Chia-HHo, Ouyang LY. The optimal pricing and ordering policy for an integrated inventory model when trade credit linked to order quantity. Applied Mathematical Modelling. Vol.33, 2978-2991, 2009

[4] Chen SC, Barron LEC, Teng JT. Retailer's economic order quantity model when the supplier off ers conditionally permissible delay in payments linked to order quantity. International Journal of Production Economics. Vol. 155, 284-291, 2014

[5] Chung KJ, Goyal SK, Huang YF. The optimal inventory policies under permissible delay in payments depending on the ordering quantity. International Journal of production Economics. Vol.95, No.2, 203-213, 2005

[6] Chung KJ, Liao J J. Lot-sizing decisions under trade credit depending on the ordering quantity. Computers and Operations Research. Vol.31, No. 6,909-928, 2004

[7] Chung KJ, Liao J J. The optimal ordering policy of the EOQ model under trade credit depending on the ordering quantity from the DCF approach. European journal of Operations Research. Vol.196, No.2, 563-568, 2009

[8] Chung, KJ, Hung CH, Dye CY. An inventory model for deteriorating items with linear trend demand under the condition of permissible delay in payments. Production planning and control. Vol.12, 274-282, 2001

[9] Chung KJ, Chang SL, Yang WD. The optimal cycle time for exponential deteriorating products under trade credit financing. The Engineering Economist. Vol.46, 232-42, 2001

[10] De LN, Goswami A. Probabilistic EOQ model for deteriorating items under trade credit financing. International Journal of System Science. Vol.40, No.4, 335-346, 2009

[11] Goyal SK. Economic order quantity under condition of permissible delay in payments. Journal of operation
Research Society. Vol.36, 335-338, 1985

[12] Huang YF. Optimal retailer's ordering policies in the EOQ model under trade credit financing. Journal of the Operational Research Society. Vol.54, 1011-1015, 2003

[13] Hwang H, Shinn SW. Retailer's pricing and lot sizing policy for exponentially deteriorating product under the condition of permissible delay in payments. Computers and Operations Research. Vol.24,539-547, 1997

[14] Jamal AMM, Sarker BR, Wang S. A ordering policy for deteriorating items with allowable shortages and permissible delay in payment. Journal of the Operations Research Society. Vol.48, 826-833,1997

[15] Jiang W, Skouri K, Teng JT, Ouyang LY. A note on "Replenishment policies for non-instantaneous deteriorating items with price and stock sensitive demand under permissible delay in payments". International Journal of Production Economics. Vol.155, 324-329, 2014

[16] Li R, Teng JT, Zheng Y. Optimal credit term, order quantity and selling price for perishable product when demand depends on selling price, expiration date and credit period. Annals of operations Research. Vol.280, 377-405, 2019

[17] Mahato G C. An EPQ-based model for exponentially deteriorating items under retailer partial trade credit policy in supply chain. Expert system with applications. Vol.39, No.3, 3537-3550, 2012

[18] Musa A, Sani B. Inventory ordering policies of delayed decorating items under permissible delay in payments. International Journal of Production Economics. Vol.136, No.1, 75-83, 2012

[19] Pramanick P, Maity MK. A note on "Replenishment policies for non-instantaneous deteriorating items with price and stock sensitive demand under permissible delay in payments". Engineering Application of Artificial Intelligence. Vol.85, 194-207, 2019

[20] Sarker BR, Jamal AMM, Wang S. Optimal payment time under permissible delay in payment for products with deterioration. Production Planning and Control. Vol.11, 380-390, 2001

[21] Shah NH, Shah YK. A lot size model for exponentially deteriorating inventory when delay in payments is permissible. Cahiers du CERO, Belgium. Vol 35, 1-9, 1993

[22] Shah NH. Probabilistic time scheduling model for exponentially decaying inventory when delay in payments is permissible. International Journal of Production Economics. Vol.32, 77-82,1993

[23] Shah NH, Shah YK. A discrete in time Probabilistic inventory model for deteriorating items under conditions of permissible delay in payments. International Journal of System science. Vol.29,121-125, 1998

[24] Shah VR, Patel NC, Shah DK. Economic ordering quantity when delay in payments of order and shortages are permitted. Gujarat Statistical Review. Vol.15, No.2, 51-56, 1998

[25] Teng JT, Yang HL. An Inventory model for increasing demand under two levels of trade credit linked to order quantity. Applied Mathematical ModellingVol.37,7624-76 32,2013 
[26] Tiwari S, Barron LEC, Shaikh AA, Choh M. Retailer's optimal ordering policy for deteriorating items under order size dependent trade credit and complete backlogging.
Computers and Industrial Engineering. Vol.139, Article 105559, 2020 